\title{
Cubic curves and totally geodesic subvarieties of moduli space
}

\section{Citation}

McMullen, Curtis, Ronen Mukamel, and Alex Wright. 2017. "Cubic Curves and Totally Geodesic Subvarieties of Moduli Space." Annals of Mathematics 185 (3) (May 1): 957-990. doi:10.4007/ annals.2017.185.3.6.

\section{Published Version}

doi:10.4007/annals.2017.185.3.6

\section{Permanent link}

http://nrs.harvard.edu/urn-3:HUL.InstRepos:34334609

\section{Terms of Use}

This article was downloaded from Harvard University's DASH repository, and is made available under the terms and conditions applicable to Open Access Policy Articles, as set forth at http:// nrs.harvard.edu/urn-3:HUL.InstRepos:dash.current.terms-of-use\#OAP

\section{Share Your Story}

The Harvard community has made this article openly available.

Please share how this access benefits you. Submit a story.

Accessibility 


\title{
Cubic curves and totally geodesic subvarieties of moduli space
}

\author{
Curtis T. McMullen, Ronen E. Mukamel and Alex Wright
}

16 March 2016

\section{Contents}

1 Introduction . . . . . . . . . . . . . . . . 1

2 Cubic curves ..................... 6

3 The flex locus . . . . . . . . . . . . . . . 11

4 The gothic locus . . . . . . . . . . . . . . . 14

$5 \quad F$ is totally geodesic .................. 19

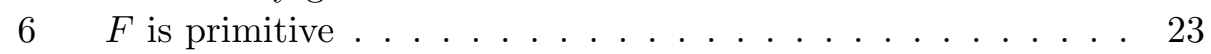

$7 \quad$ The Kobayashi metric on $F \ldots \ldots \ldots \ldots$

8 Teichmüller curves in $\mathcal{M}_{4} \ldots \ldots \ldots \ldots$. . . . . . . 27

9 Explicit polygonal constructions . . . . . . . . . . . . . 29 


\section{Introduction}

Let $\mathcal{M}_{g, n}$ denote the moduli space of compact Riemann surfaces of genus $g$ with $n$ marked points. A complex geodesic is a holomorphic immersion $f: \mathbb{H} \rightarrow \mathcal{M}_{g, n}$ that is a local isometry for the Kobayashi metrics on its domain and range. It is known that $\mathcal{M}_{g, n}$ contains a complex geodesic through every point and in every possible direction.

We say a subvariety $V \subset \mathcal{M}_{g, n}$ is totally geodesic if every complex geodesic tangent to $V$ is contained in $V$. It is primitive if it does not arise from a simpler moduli space via a covering construction.

A Teichmüller curve is a totally geodesic subvariety of $\mathcal{M}_{g, n}$ of dimension one. These rare and remarkable objects are closely related to billiards in polygons, Jacobians with real multiplication, and dynamical rigidity. They are uniformized by Fuchsian groups defined over number fields, but they are generally not arithmetic.

This paper gives the first example of a primitive, totally geodesic $T e$ ichmüller surface in moduli space. We also obtain a new infinite series of Teichmüller curves in $\mathcal{M}_{4}$, and new $\mathrm{SL}_{2}(\mathbb{R})$-invariant subvarieties in moduli spaces of quadratic differentials and holomorphic 1-forms.

Our constructions depend in a fundamental way on the classic subject of cubic curves in the plane $(\S 2)$ and space curves of genus four $(\S 4)$, giving an unexpected connection between algebraic geometry and Teichmüller theory.

The flex locus $\boldsymbol{F} \subset \mathcal{M}_{\mathbf{1}, \mathbf{3}}$. A point in $\mathcal{M}_{1,3}$ is specified by a pair $(A, P)$ consisting of a compact Riemann surface $A$ of genus 1 , and an unordered set $P \subset A$ with $|P|=3$. We can also regard $P$ as an effective divisor of degree three.

The flex locus $F \subset \mathcal{M}_{1,3}$ is defined by:

$$
F=\left\{\begin{array}{ll}
\exists \text { a degree } 3 \text { rational map } \pi: A \rightarrow \mathbb{P}^{1} \text { such that } \\
(A, P): & \text { (i) the fibers of } \pi \text { are linearly equivalent to } P \text {, and } \\
& \text { (ii) } P \text { is a subset of the cocritical points of } \pi .
\end{array}\right\}
$$

Here $x^{\prime} \in A$ is a cocritical point of $\pi$ if

$$
\left\{x, x^{\prime}\right\}=(\text { a fiber of } \pi)
$$

for some critical point $x$ of $\pi$. (We allow $x^{\prime}=x$.)

We refer to $F$ as the flex locus because, when $A$ is defined by a homogeneous cubic polynomial $f, \pi$ is given by projection from a point where $\operatorname{det} D^{2} f=0$. See $\S 3$.

The main result of this paper, proved in $\S 5$, is: 
Theorem 1.1 The flex locus $F$ is a totally geodesic, irreducible complex surface in $\mathcal{M}_{1,3}$.

Let $\mathcal{T} F \rightarrow F$ denote an irreducible component of the preimage of $F$ in $\mathcal{T}_{1,3}$. Since $\mathcal{T} F$ is totally geodesic, it is a smooth, contractible complex manifold. In $\S 6$ and $\S 7$ we will show:

Theorem 1.2 The complex manifold $\mathcal{T} F$ is not isomorphic to any traditional Teichmüller space $\mathcal{T}_{g, n}$.

Corollary 1.3 The Teichmüller surface $F \subset \mathcal{M}_{1,3}$ is primitive, i.e. it does not arise from a covering construction.

Strata. The surface $F$ is closely related to an algebraic threefold $G \subset \mathcal{M}_{4}$, which is abundantly populated by new Teichmüller curves. Our proof of Theorem 1.1 depends on this relation.

To define $G$, we first need notation for strata. Let $\Omega \mathcal{M}_{g} \rightarrow \mathcal{M}_{g}$ denote the moduli space of holomorphic 1-forms of genus $g$. As usual, given $a_{i}>0$ with $\sum_{1}^{n} a_{i}=2 g-2$, we let $\Omega \mathcal{M}_{g}\left(a_{1}, \ldots, a_{n}\right) \subset \Omega \mathcal{M}_{g}$ denote the stratum consisting of 1 -forms $(X, \omega)$ such that

$$
(\omega)=\sum_{1}^{n} a_{i} p_{i}
$$

for some distinct points $p_{1}, \ldots, p_{n} \in X$. We use exponential notation for repeated indices; for example, $\Omega \mathcal{M}_{g}(2,2, \ldots, 2)=\Omega \mathcal{M}_{g}\left(2^{g-1}\right)$.

Within a given stratum, we can impose the additional condition that there exists an involution $J: X \rightarrow X$, with fixed points $\left(p_{1}, \ldots, p_{n}\right)$, such that $J^{*}(\omega)=-\omega$. The resulting locus is a Prym stratum

$$
\Omega \mathcal{M}_{g}\left(a_{1}, \ldots, a_{n}\right)^{-} \subset \Omega \mathcal{M}_{g} .
$$

Note that $J$ is uniquely determined by $\omega$, provided $g>1$. We allow $a_{i}=0$, to account for fixed points that are not zeros.

There is a natural action of $\mathrm{SL}_{2}(\mathbb{R})$ on $\Omega_{\mathcal{M}}$ that preserves both types of strata, and whose orbits project to complex geodesics in $\mathcal{M}_{g}$.

The gothic locus $\boldsymbol{G} \subset \mathcal{M}_{\mathbf{4}}$. Given a Riemann surface $X$ with a distinguished involution $J$, we say a holomorphic map $p: X \rightarrow B$ is odd if there exists an involution $j \in \operatorname{Aut}(B)$ such that $p(J(x))=j(p(x))$ for all $x \in X$. Let

$$
\Omega G=\left\{(X, \omega) \in \Omega \mathcal{M}_{4}\left(2^{3}, 0^{3}\right)^{-}: \begin{array}{l}
\exists \text { a curve } B \in \mathcal{M}_{1} \text { and an odd }, \\
\text { degree } 3 \text { rational map } p: X \rightarrow B \\
\text { such that }|p(Z(\omega))|=1 .
\end{array}\right\}
$$


Here $Z(\omega)$ denotes the zero set of $\omega$. The condition that $p$ sends the three zeros of $\omega$ to a single point implies that $p_{*}(\omega)=0$.

We refer to the variety $G$ obtained by projecting $\Omega G$ to $\mathcal{M}_{4}$ as the gothic locus. (The terminology is inspired by Figure 1.)

The relationship between $F$ and $G$ can be summarized as follows: given any form $(X, \omega) \in \Omega G$ with involution $J$, we obtain a point $(A, P) \in F$ by setting $(A, q)=\left(X, \omega^{2}\right) / J$, and marking the poles of $q$.

Using this natural map $\Omega G \rightarrow F$, in $\S 4$ we will show:

Theorem 1.4 The space $\Omega G \subset \Omega \mathcal{M}_{4}$ is a closed, irreducible variety of dimension 4, locally defined by real linear equations in period coordinates.

In particular, the variety $\Omega G$ is locally isomorphic to a finite union of $4-$ dimensional subspaces of $\mathbb{C}^{10}$.

Corollary 1.5 The locus $\Omega G$ is invariant under the natural action of $\mathrm{SL}_{2}(\mathbb{R})$.

The crux of the proof of Theorem 1.4 is a lower bound on $\operatorname{dim} \Omega G$ coming from our study of $F$. The surprise is that a small number of conditions on the periods of $\omega$ produce an elliptic curve $B$ and a map $p: X \rightarrow B$.

The fact that $F$ is totally geodesic follows readily from Corollary 1.5 , by transporting $\mathrm{SL}_{2}(\mathbb{R})$-orbits in $\Omega G$ to complex geodesics in $F$.

Teichmüller curves and real multiplication. Let $\mathcal{O}_{D} \cong \mathbb{Z}[(D+\sqrt{D}) / 2]$ denote the real quadratic order of discriminant $D$, where $D>0$ and $D=0$ or $1 \bmod 4$.

Let $(X, \omega)$ be a form whose membership in $\Omega G$ is ratified by an involution $J$ and a map $p: X \rightarrow B$. Then we also have a natural map $\phi: X \rightarrow A=$ $X / J$. Taking the quotient of $\operatorname{Jac}(X)$ by divisors pulled back from $A$ and $B$, we obtain the polarized Abelian surface

$$
C=C(X \mid A, B)=\operatorname{Jac}(X) / \operatorname{Im}(\operatorname{Jac}(A) \times \operatorname{Jac}(B)) .
$$

Let $\Omega G_{D} \subset \Omega G$ denote the locus where $C$ admits real-multiplication by $\mathcal{O}_{D}$ with $\omega$ as an eigenform. Its projection to $\mathcal{M}_{4}$ will be denoted by $G_{D}$. In $\S 8$ we will show:

Theorem 1.6 For every discriminant $D>0$, the locus $G_{D} \subset \mathcal{M}_{4}$ is a finite union of Teichmüller curves.

Theorem 1.7 If $D$ is not a square, then every component of $G_{D}$ is geometrically primitive. 
Theorem 1.8 If the stabilizer of a form in $\Omega G$ contains a hyperbolic element $\gamma$, then $\operatorname{SL}(X, \omega)$ is a lattice and $(X, \omega) \in \Omega G_{D}$ for some $D$ with $\mathbb{Q}(\sqrt{D})=\mathbb{Q}(\operatorname{tr} \gamma)$.
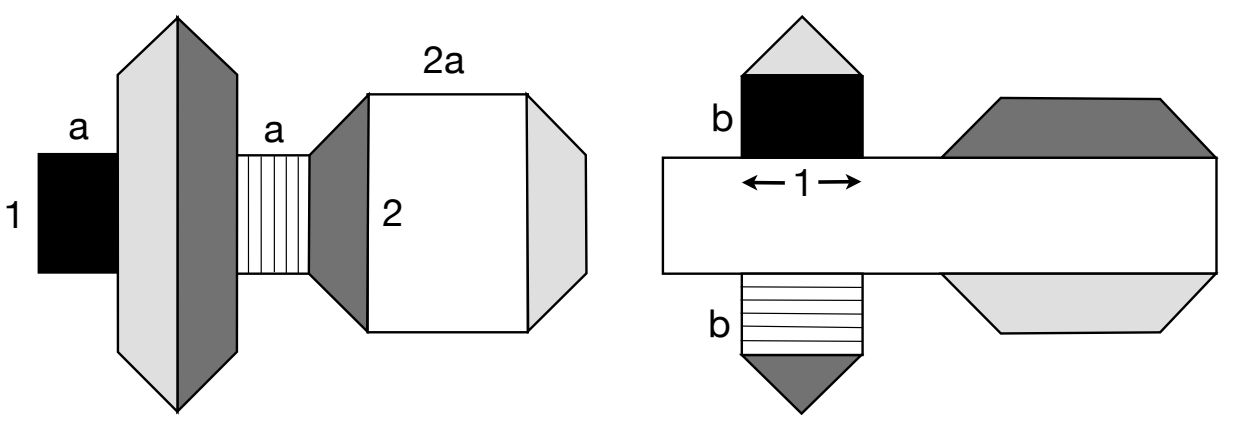

Figure 1. The cathedral polygon $P(a, b)$.

Polygon models for gothic forms. To conclude, we will describe an explicit construction of forms in the locus $\Omega G$.

Figure 1 illustrates two copies of a polygon $P(a, b) \subset \mathbb{C}$. This polygon is symmetric about the $x$-axis, and each of its edges has slope $0, \infty$ or \pm 1 . Pairs of parallel edges are glued together by translation to form a compact Riemann surface $X=P(a, b) / \sim$ of genus 4 . The edge pairings for $P(a, b)$ can be read off from the condition that regions with the same shade on the right or the left cover cylinders on $X$. The form $d z \mid P(a, b)$ descends to a form $\omega \in \Omega(X)$ with three double zeros, coming from the vertices of $P(a, b)$.

In $\S 9$ we will show:

Theorem 1.9 For any $a, b>0$, the holomorphic 1-form

$$
(X, \omega)=(P(a, b), d z) / \sim
$$

lies in $\Omega G$.

Theorem 1.10 If, in addition, there are rational numbers $x, y$ and $d \geq 0$ such that

$$
a=x+y \sqrt{d}, \quad b=-3 x-3 / 2+3 y \sqrt{d},
$$

then $(X, \omega)$ generates a Teichmüller curve. In fact $(X, \omega) \in G_{D}$ for some $D$ with $\mathbb{Q}(\sqrt{D})=\mathbb{Q}(\sqrt{d})$. 
Corollary 1.11 Every real quadratic field $K$ arises as $K=\mathbb{Q}(\sqrt{D})$ for some $D$ with $G_{D} \neq \emptyset$.

\section{Outline of the paper.}

1. In $\S 2$ and $\S 3$ we describe the surface $F$ from the perspective of classical projective geometry.

Every pair $(A, P) \in \mathcal{M}_{1,3}$ can be presented as a triple of collinear points on a smooth cubic curve in the plane,

$$
P=L \cap A \subset \mathbb{P}^{2} \text {. }
$$

Similarly, every degree three rational map $\pi_{S}: A \rightarrow \mathbb{P}^{1}$, with fibers linearly equivalent to $P$, is obtained by projection from a point $S \in \mathbb{P}^{2}$. We find that $\pi_{S}$ has a triple of collinear cocritical points if and only if $S$ itself lies on a related cubic curve, the Hessian $H A \subset \mathbb{P}^{2}$.

Since the moduli space $\mathcal{M}_{1}$ of smooth cubics is 1-dimensional, this shows that $F$ itself is 2 -dimensional. In fact, $F$ is naturally swept out by an open subset $\widetilde{F}$ of the universal Cayleyan, a smooth surface discussed in $\S 3$.

2. In $\S 4$ we use the fact that $\operatorname{dim} F=2$ to show $\operatorname{dim} \Omega G=4$; while in $\S 5$, we show that in period coordinates, $\Omega G$ is contained in a finite union of 4 -dimensional linear spaces defined over $\mathbb{R}$. It follows that $\Omega G$ is $\mathrm{SL}_{2}(\mathbb{R})$-invariant and that $F$ is totally geodesic.

The algebraic formula for $G$ given in equation (4.7) below also provides a direct proof that $\operatorname{dim} \Omega G \geq 4$.

3. In $\S 6$ we review the theory of covering constructions, and exhibit another totally geodesic surface $S_{11} \subset \mathcal{M}_{1,3}$ which arises in this way. We then give a topological proof that $F$ is primitive.

4. In $\S 7$ we show, via an analysis of the Kobayashi metric, that $\mathcal{T} F$ is not isomorphic to any traditional Teichmüller space $\mathcal{T}_{g, n}$. This result gives a geometric proof that $F$ is primitive, and suggest that one might regard $\mathcal{T} F$ itself as a new type of Teichmüller space, on an equal footing with $\mathcal{T}_{g, n}$.

5. In $\S 8$ we show that the loci $G_{D} \subset \mathcal{M}_{4}$ are finite unions of Teichmüller curves; the proof is similar to the case of the Weierstrass curves in $\mathcal{M}_{2}$ [Mc1]. Finally, in $\S 9$ we show that Figure 1 defines 1 -forms in $\Omega G$ and, for suitable parameters, these forms generate Teichmüller curves in $\bigcup G_{D}$. 
Notes and references. The components of $G_{D}$ with $\sqrt{D}$ irrational give a new, infinite series of geometrically primitive Teichmüller curves.

The previously known examples consist of four infinite series and two sporadic cases. The first three series come from the Weierstrass curves $W_{D} \subset \mathcal{M}_{g}$, defined for $g=2,3$ and 4 [Ca], [Mc1], [Mc3]. The fourth is the Bouw-Möller series, which gives finitely many more examples in $\mathcal{M}_{g}$ for every $g>1[\mathrm{BM}]$; see also $[\mathrm{V}],[\mathrm{Ho}],[\mathrm{Wr} 1]$. Finally, there are 2 sporadic examples associated to the Coxeter diagrams $E_{7}$ and $E_{8}$; see [KS], [Vo] and [Lei].

The locus $\Omega G$ itself has many interesting properties. For example, it is the first known primitive, $\mathrm{SL}_{2}(\mathbb{R})$-invariant subvariety of $\Omega \mathcal{M}_{g}$ defined over $\mathbb{Q}$ (in period coordinates), aside from the obvious examples like strata. For more on the properties of affine invariant manifolds such as $\Omega G$, see [Wr2] and [Wr3].

A program provided by A. Eskin led us to focus on the cathedral forms and provided evidence that they should lie in a new invariant subvariety of $\Omega \mathcal{M}_{4}$. A special case of Theorem 1.10 was first proved using the algorithm described in [Mu1], which showed directly that $\operatorname{SL}(X, \omega)$ is a lattice for $(x, y, d)=(0,1 / 2,2)$.

Further results and useful background can be found in the surveys [Mas], [Mo3] and [Z].

Acknowledgements. We would like to thank I. Dolgachev, A. Eskin, M. Mirzakhani and A. Patel for useful discussions.

\section{Cubic curves}

In this section we recall some classical constructions from projective geometry. These constructions associate, to any smooth plane cubic curve $A \subset \mathbb{P}^{2}$, three other curves: the Hessian $H A \subset \mathbb{P}^{2}$, the Cayleyan $C A$, and the satellite Cayleyan $S A$. The last two reside in the dual projective plane $\check{\mathbb{P}}^{2}$. In the next section, we will see that the points in the flex locus lying over $A$ are naturally parameterized by $S A$.

Useful references for this material include [Cay], [Cr], [Sal] and [Dol].

Plane cubics. Let $A$ be a plane cubic curve, given as the zero set

$$
A=Z(f) \subset \mathbb{P}^{2}
$$

of a homogeneous polynomial $f: \mathbb{C}^{3} \rightarrow \mathbb{C}$ of degree 3 . We say $A$ is a triangle if it is projectively equivalent to the cubic $Z(X Y Z)$, and a Fermat cubic if 
it is equivalent to $Z\left(X^{3}+Y^{3}+Z^{3}\right)$. We will be mostly interested in the case where $f$ is irreducible and $A$ is smooth.

Polars, satellites and projections. Let $S=[s]$ denote the point in $\mathbb{P}^{2}$ determined by a nonzero vector $s=\left(s_{0}, s_{1}, s_{2}\right) \in \mathbb{C}^{3}$. The polar of $A$ with respect to $S$ is defined by

$$
\operatorname{Pol}(A, S)=Z(\langle s, \nabla f(x)\rangle)=Z\left(\sum s_{i} \frac{d f}{d x_{i}}\right) .
$$

The satellite conic of $A$ (cf. [Sal, p. 62]) is defined by

$$
\operatorname{Sat}(A, S)=Z\left(\langle x, \nabla f(s)\rangle^{2}-4 f(s)\langle s, \nabla f(x)\rangle\right) .
$$

Projection from $S$ defines a rational map

$$
\pi_{S}: A \rightarrow \mathbb{P}^{1} .
$$

(Intrinsically, the target is the linear system of hyperplanes through $S$.) If $A$ is smooth and $S \notin A$, then projection from $S$ is a smooth cubic map, and one can readily check that

$$
A \cap \operatorname{Pol}(A, S)=\left\{\text { critical points of } \pi_{S}\right\} .
$$

Moreover, the cocritical points of $\pi_{S}$ (defined by equation (1.1)) come from its satellite: we have

$$
A \cap \operatorname{Sat}(A, S)=\left\{\text { cocritical points of } \pi_{S}\right\} .
$$

Note. Relation (2.3) holds, more generally, for any smooth hypersurface $A \subset \mathbb{P}^{n}$, and relation (2.4) holds whenever $A$ is cubic, as does the alternative formula:

$$
\operatorname{Pol}(A, S)=Z\left(\left\langle D^{2} f(s) x, x\right\rangle\right) .
$$

Lattès maps. For the remainder of this section, we assume that the cubic curve $A=Z(f)$ is smooth. The tangent line to $A$ at $x$ will be denoted by $T_{x} A \subset \mathbb{P}^{2}$. The space of tangent lines forms the dual sextic

$$
\check{A}=\left\{T_{x} A: x \in A\right\} \subset \check{\mathbb{P}}^{2}
$$

in the dual projective plane. Let $\left\{x, x^{\prime}\right\}$ denote the points where $T_{x} A$ meets $A$. If a line $L$ meets $A$ at $(a, b, c)$, then the three points $\left(a^{\prime}, b^{\prime}, c^{\prime}\right)$ also lie on a line $L^{\prime}=\delta_{A}(L)$. This construction defines the holomorphic Lattès map

$$
\delta_{A}: \check{\mathbb{P}}^{2} \rightarrow \check{\mathbb{P}}^{2}
$$


associated to $A$, of interest in complex dynamics (see e.g. [DH], [Ro], [Be]). Its algebraic degree is 4 .

The Hessian. The Hessian of $A$ is the cubic curve defined by $H A=$ $Z\left(\operatorname{det} D^{2} f\right)$. The nine flexes of $A$ are given by $H A \cap A$.

The Hessian can be described geometrically in terms of the polars and satellites of $A$; namely,

$$
H A=\left\{S \in \mathbb{P}^{2}: \text { the polar conic } \operatorname{Pol}(A, S) \text { is singular }\right\},
$$

and

$$
A \cup H A=\left\{S \in \mathbb{P}^{2}: \text { the satellite conic } \operatorname{Sat}(A, S) \text { is } \operatorname{singular}\right\} .
$$

These statements follow directly from equations (2.5) and (2.2). The second implies:

For $S \notin A$, the projection $\pi_{S}: A \rightarrow \mathbb{P}^{1}$ has three collinear cocritical points $\Longleftrightarrow S \in H A$.

These three cocritical points are distinct, provided $S$ is not on the line through a flex of $A$; in particular:

$$
\text { A generic line } L \in S A \text { meets A transversely. }
$$

The Cayleyan. For each $S \in H A$ we have a pair of distinct lines such that

$$
\operatorname{Pol}(A, S)=L_{1} \cup L_{2} .
$$

The Cayleyan $C A \subset \check{\mathbb{P}}^{2}$ is the set of all lines that arise in this way; that is,

$$
C A=\left\{L \in \check{\mathbb{P}}^{2}: L \subset \operatorname{Pol}(A, S) \text { for some } S \in H A\right\} .
$$

The point $S$ is uniquely determined by $L$, since it lies on $T_{x} A$ for all $x \in L \cap A$. Thus we have a natural degree two covering map, $C A \rightarrow H A$. The curve $C A$ is also cubic; see equation (2.14) below.

The satellite Cayleyan. Note that if $S \notin A$ and $x \in A$ is a critical point of $\pi_{S}$, then $x^{\prime}$ is a cocritical point. As a consequence, if $S \in H A$ and $\operatorname{Pol}(A, S)=L_{1} \cup L_{2}$, then

$$
\operatorname{Sat}(A, S)=L_{1}^{\prime} \cup L_{2}^{\prime}
$$

We refer to the set of lines which arise in this way as the satellite Cayleyan,

$$
S A=\delta_{A}(C A)=\left\{L \in \check{\mathbb{P}}^{2}: L \subset \operatorname{Sat}(A, S) \text { for some } S \in H A\right\} .
$$




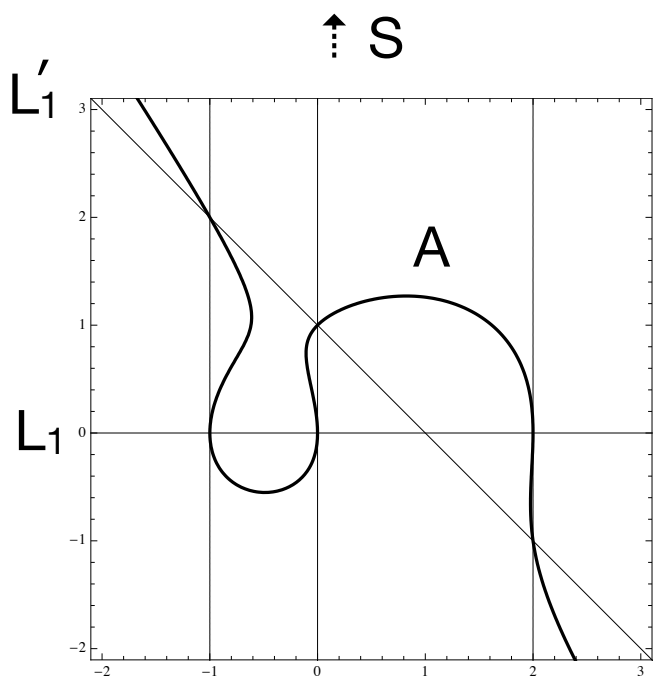

Figure 2. We have $L_{1} \subset \operatorname{Pol}(A, S)$ and $L_{1}^{\prime} \subset \operatorname{Sat}(A, S)$.

Since $C A$ is irreducible, so is $S A$. It is generically a curve of degree 12 and genus one, with interesting singularities.

Normal form. Here is an explicit description of the polar and satellite lines for an arbitrary smooth cubic $A \subset \mathbb{P}^{2}$ as seen from a point $S \in H A-A$.

Let $\operatorname{Pol}(A, S)=L_{1} \cup L_{2}$ and $\operatorname{Sat}(A, S)=L_{1}^{\prime} \cup L_{2}^{\prime}$. It is easy to see that $S \notin L_{1}$. Choose affine coordinates $(x, y)$ on $\mathbb{C}^{2} \subset \mathbb{P}^{2}$ so that $S=[0: 1: 0]$ is the vertical point at infinity, and $L_{1}$ is the $x$-axis. Then, it is readily verified that $A$ is defined by a cubic equation of the form

$$
f(x, y)=y^{3}+b(x) y^{2}+c(x)=0
$$

where $b, c \in \mathbb{C}[x]$ are polynomials of degrees (at most) 1 and 3 respectively; and that the polar and satellite lines for any such cubic are defined by the vanishing of the linear forms:

$$
\begin{array}{ll}
L_{1}(x, y)=y ; & L_{2}(x, y)=y+(2 / 3) b(x) ; \quad \text { and } \\
L_{1}^{\prime}(x, y)=y+b(x) ; & L_{2}^{\prime}(x, y)=y-(1 / 3) b(x) .
\end{array}
$$

In particular, all four lines pass through a single point.

Figure 2 shows the lines $L_{1}$ and $L_{1}^{\prime}$ for the cubic defined by equation (2.10) with $b(x)=x-1$ and $c(x)=x(x+1)(x-2)$. 
The Hesse pencil. Finally we discuss the Hesse pencil of cubics. A useful reference for this topic is $[\mathrm{AD}]$.

The Hesse pencil is the family of cubic curves $A_{t} \subset \mathbb{P}^{2}$, defined for $t \in \mathbb{P}^{1}$ by

$$
X^{3}+Y^{3}+Z^{3}-3 t X Y Z=0 .
$$

The curve $A_{0}$ is a Fermat cubic, while $A_{\infty}$ is the triangle defined by $X Y Z=$ 0 . The cubic $A_{t}$ is smooth over the points

$$
\widetilde{\mathcal{M}}_{1}=\left\{t \in \mathbb{C}: t^{3} \neq 1\right\} \subset \mathbb{P}^{1}
$$

otherwise it is a triangle.

The base locus $E=A_{0} \cap A_{\infty}$ of the Hesse pencil coincides with the nine flexes of $A_{0}$, as well as the flexes of every other smooth curve in the family. Thus, we can regard an element of the Hesse pencil as an elliptic curve with marked 3-torsion. More precisely, we have a natural isomorphism

$$
\widetilde{\mathcal{M}}_{1} \cong \mathbb{H} / \Gamma(3)
$$

the modular $j$-invariant of $A_{t}$ is given by

$$
j(t)=\frac{27\left(t^{4}+8 t\right)^{3}}{\left(t^{3}-1\right)^{3}}
$$

and $j$ defines a covering map of orbifolds

$$
j: \widetilde{\mathcal{M}}_{1} \rightarrow \mathcal{M}_{1}=\mathbb{H} / \mathrm{SL}_{2}(\mathbb{Z}) \cong \mathbb{C},
$$

with deck group $\mathrm{PSL}_{2}\left(\mathbb{F}_{3}\right) \cong A_{4}$. Note that $A_{t}$ is a Fermat cubic iff $j(t)=0$, which gives $t=0,-2$ or $1 \pm \sqrt{-3}$.

When $A$ belongs to the Hesse pencil, so do the cubics $H A$ and $C A$ (using the natural dual basis to identify $\mathbb{P}^{2}$ and $\check{\mathbb{P}}^{2}$ ). In fact, $H A_{t}=A_{h}$ and $C A_{t}=A_{c}$ for the values

$$
h(t)=\frac{4-t^{3}}{3 t^{2}} \text { and } \quad c(t)=\frac{2+t^{3}}{3 t}
$$

(see $[\mathrm{AD}, \S 3]$ ). Using these formulas one can verify that, for a smooth cubic curve $A$ :

Either $A$ is a Fermat cubic and $H A$ and $C A$ are triangles, or $H A$ and $C A$ are also smooth cubics. 


\section{The flex locus}

In this section we discuss the flex locus from the perspective of plane cubics. We begin by establishing an alternative definition of $F$, using the language of $\S 2$.

Theorem 3.1 $A$ point $(A, P) \in \mathcal{M}_{1,3}$ lies in $F$ if and only if there is a plane cubic model for $A$ and a line $L$ in the satellite Cayleyan $S A$ such that $P=A \cap L$.

Using the universal Cayleyan, we then show:

Theorem 3.2 The flex locus $F \subset \mathcal{M}_{1,3}$ is the image of a smooth, irreducible surface under a proper immersion.

Finally we define a 4-dimensional bundle of quadratic differentials $Q F \rightarrow F$, analogous to $\Omega G \rightarrow G$, and show:

Theorem 3.3 The locus $Q F \subset Q \mathcal{M}_{1,3}$ is a closed, irreducible subvariety of dimension four.

Markings. To prove Theorems 3.2 and 3.3 we will explicitly construct a smooth, irreducible surface $\widetilde{F}$, a finite manifold cover of moduli space

$$
u: \widetilde{\mathcal{M}}_{1,3} \rightarrow \mathcal{M}_{1,3}
$$

and a proper immersion

$$
\delta: \widetilde{F} \rightarrow \widetilde{\mathcal{M}}_{1,3},
$$

such that $u \circ \delta$ sends $\widetilde{F}$ to $F$.

The smooth surface $\widetilde{F}$ is of interest in its own right. A point in $\widetilde{F}$ corresponds to triple $\left(A, P, \pi_{S}\right)$, with $S \in H A$, satisfying the definition of $F$ in $\S 1$, together with a marking of the 3 -torsion of $A$. The surface $F$ itself is not smooth, and these choices serve to separate its sheets and resolve its orbifold points.

Cubic models. To make the connection to $\S 2$, recall that any Riemann surface $A \in \mathcal{M}_{1}$ can be presented as a smooth cubic curve $A \subset \mathbb{P}^{2}$. This plane cubic model for $A$ is unique up to automorphisms of $A$ and $\mathbb{P}^{2}$.

Proof of Theorem 3.1. Suppose $(A, P) \in F$. Let $A \subset \mathbb{P}^{2}$ be the plane cubic model determined by the complete linear system $|P|$. Then $P=A \cap L$ for some line $L \in \check{\mathbb{P}}^{2}$. Since $(A, P) \in F$, there is a degree three rational map 
$\pi: A \rightarrow \mathbb{P}^{1}$ such that (i) the fibers of $\pi$ are linearly equivalent to $P$ and (ii) $P$ is contained in the cocritical points of $\pi$. Property (i) implies that $\pi$ is given by projection from some point $S \in \mathbb{P}^{2}-A$; and (ii) implies that $P$ is contained in the satellite conic $\operatorname{Sat}(A, S)$ (see assertion (2.4)). Since $P$ consists of 3 distinct points, this implies we have $L \subset \operatorname{Sat}(A, S)$; hence the satellite conic is singular, and we have $L \in S A$. The converse is similar.

Cubics and lines. We now turn to the proof of Theorem 3.2. For concreteness, we will work with the family of Hesse cubics $A_{t} \subset \mathbb{P}^{2}$ defined by (2.12). Consider the Zariski open subset of $\mathbb{P}^{1} \times \check{\mathbb{P}}^{2}$ defined by

$$
\widetilde{\mathcal{M}}_{1,3}=\left\{(t, L): A_{t} \text { is smooth and }\left|L \cap A_{t}\right|=3\right\} \text {. }
$$

Since every cubic occurs, up to isomorphism, in the Hesse family, we have a natural covering map of orbifolds $u: \widehat{\mathcal{M}}_{1,3} \rightarrow \mathcal{M}_{1,3}$ given by

$$
u(t, L)=\left(A_{t}, L \cap A_{t}\right) .
$$

We remark that the deck group $\Gamma$ of $\widetilde{\mathcal{M}}_{1,3} / \mathcal{M}_{1,3}$ has order 216 ; it satisfies

$$
\Gamma \cong \operatorname{Aut}\left(\mathbb{P}^{2}\right)^{E} \cong \mathbb{F}_{3}^{2} \ltimes \mathrm{SL}_{2}\left(\mathbb{F}_{3}\right)
$$

where $\operatorname{Aut}\left(\mathbb{P}^{2}\right)^{E}$ denotes the group of projective transformations stabilizing nine basepoints $E=A_{0} \cap A_{\infty}$ of the Hesse pencil.

The universal Cayleyan. Next we define the universal Cayleyan over the Hesse family by

$$
C A_{\mathbb{P}^{1}}=\left\{(t, L) \in \mathbb{P}^{1} \times \check{\mathbb{P}}^{2}: L \in C A_{t}\right\}
$$

The locus $C A_{\mathbb{P}^{1}} \rightarrow \mathbb{P}^{1}$ is smooth elliptic surface, even though some of its fibers, such as $C A_{0}$, are triangles. In particular, $C A_{\mathbb{P}^{1}}$ is irreducible. Smoothness follows from the fact that $c(t)$ has a simple pole in equation (2.14), so that near a triangular fiber $C A_{\mathbb{P}^{1}}$ is locally isomorphic to the surface $x y=t$.

Lattès maps. Recall from equation (2.13) that $A=A_{t}$ is smooth iff $t \in \widetilde{\mathcal{M}}_{1} \subset \mathbb{P}^{1}$. Let

$$
\delta: \widetilde{\mathcal{M}}_{1} \times \check{\mathbb{P}}^{2} \rightarrow \widetilde{\mathcal{M}}_{1} \times \check{\mathbb{P}}^{2}
$$

be the proper map defined by $\delta(t, L)=\left(t, \delta_{A_{t}}(L)\right)$ using the Lattès construction (2.6). 
Normalization of a cover of $\boldsymbol{F}$. Finally we define a Zariski open subset of the universal Cayleyan by

$$
\widetilde{F}=C A_{\mathbb{P}^{1}} \cap \delta^{-1}\left(\widetilde{\mathcal{M}}_{1,3}\right) .
$$

By observation (2.9), $\widetilde{F}$ is nonempty; and since $C A_{\mathbb{P}^{1}}$ is a smooth, irreducible surface, so is $\widetilde{F}$.

Proof of Theorem 3.2. Since the map $\delta$ in equation (3.2) is proper, so is its restriction $\delta \mid \widetilde{F}$. It is also an immersion, since the critical values of $\delta_{A}$ correspond to lines with $|L \cap A|<3$, and these configurations are excluded from $\widetilde{\mathcal{M}}_{1,3}$. Since $u$ is a covering map of orbifolds, the composition $u \circ \delta$ is a proper immersion; and since $S A=\delta_{A}(C A)$, its image is $F$ by Theorem 3.1.

Corollary 3.4 The surface $F$ is birational to $\mathbb{P}^{2}$.

Proof. Since the hyperelliptic involution $-I \in \mathrm{SL}_{2}\left(\mathbb{F}_{3}\right)$ belongs to the group $\Gamma$ in equation (3.1), the map $u \circ \delta: \widetilde{F} \rightarrow F$ factors through a rational quotient of the elliptic surface $C A_{\mathbb{P}^{1}}$. Thus $F$ itself is rational.

One can also check that in the example of Figure 2, $S$ is uniquely determined by $L_{1}^{\prime} \in S A$, and hence:

The map $\delta: \widetilde{F} \rightarrow \widetilde{\mathcal{M}}_{1,3}$ is generically 1-to-1.

The space of quadratic differentials $\boldsymbol{Q F} \rightarrow \boldsymbol{F}$. We conclude by defining a bundle of quadratic differentials to record the directions of Teichmüller geodesics in $F$.

Recall that the cotangent space to a point $(Y, P) \in \mathcal{M}_{g, n}$ is naturally identified with the vector space $Q(Y, P)$ of meromorphic quadratic differentials $q$ on $Y$ with $(q)+P \geq 0$. A point in the moduli space of quadratic differentials, $Q \mathcal{M}_{g, n} \rightarrow \mathcal{M}_{g, n}$, is specified by a triple $(Y, P, q)$ as above with $q \neq 0$.

Now let $(A, P) \in \mathcal{M}_{1,3}$ be an elliptic curve with marked points whose membership in $F$ is ratified by a rational map $\pi: A \rightarrow \mathbb{P}^{1}$ of degree three. Let

$$
Q F(A, P, \pi)=\{q \in Q(A, P):(q)=Z-P \text { for some fiber } Z \text { of } \pi\} .
$$

To take into account of the possibility that $\pi$ is not unique, let $Q F(A, P)=$ $\bigcup_{\pi} Q(A, P, \pi)$. Finally, let $Q F \rightarrow F$ denote the subspace of $Q \mathcal{M}_{1,3} \rightarrow \mathcal{M}_{1,3}$ whose fiber over $(A, P) \in F$ is $Q F(A, P)$. 
Proof of Theorem 3.3. Recall that for $(t, L) \in \widetilde{F}$, there is a unique $S \in \mathbb{P}^{2}$ such that $L \subset \operatorname{Pol}\left(A_{t}, S\right)$, and a unique $L^{\prime} \subset \operatorname{Sat}\left(A_{t}, S\right)$ such that $L^{\prime}=\delta_{A_{t}}(L)$. Define a bundle $Q \widetilde{F} \rightarrow \widetilde{F}$ with fibers

$$
Q \widetilde{F}(t, L)=Q F\left(A_{t}, L^{\prime} \cap A_{t}, \pi_{S}\right) \cong \mathbb{C}^{2}-\{0\} .
$$

We then have a natural proper map immersion $Q \delta: Q \widetilde{F} \rightarrow Q \mathcal{M}_{1,3}$ covering the map $\delta: \widetilde{F} \rightarrow \mathcal{M}_{1,3}$, and its image is $Q F$. Since $\widetilde{F}$ is irreducible, so is $Q F$ and clearly $\operatorname{dim} Q F=\operatorname{dim} Q \widetilde{F}=4$.

Sheets of $\boldsymbol{F}$. The proof shows that, given $(A, P) \in F$, there are only finitely many possibilities for the associated map $\pi: A \rightarrow \mathbb{P}^{1}$, and the different choices of $\pi$ index the different sheets of the immersed surface $F$ passing through $(A, P)$.

\section{The gothic locus}

In this section we discuss the correspondence between quadratic differentials and Prym forms, and use it to relate the flex locus $F$ to the threefold $G \subset$ $\mathcal{M}_{4}$ defined in $\S 1$. We will show:

Theorem 4.1 The squaring map gives a natural algebraic isomorphism

$$
\mathrm{sq}: \Omega G \rightarrow Q F\left(-1^{3}, 1^{3}\right) .
$$

Here $Q F\left(-1^{3}, 1^{3}\right)$ denotes the intersection of $Q F$ with the principal stratum of $Q \mathcal{M}_{1,3}$.

Strata. We begin by reviewing notation for strata of quadratic differentials with marked points. Recall that a point of $Q \mathcal{M}_{g, n}$ is specified by a triple $(Y, P, q)$. The stratum:

$$
Q \mathcal{M}_{g, n}\left(a_{1}, \ldots, a_{s}\right) \subset Q \mathcal{M}_{g, n}
$$

is defined by the requirement that there exist distinct points $\left(p_{1}, \ldots, p_{s}\right)$ in $Y$ such that $P=\left\{p_{1}, \ldots, p_{n}\right\}$ and the divisor of $q$ satisfies

$$
(q)=\sum_{1}^{s} a_{i} p_{i} .
$$

Here $\sum a_{i}=4 g-4, a_{i} \geq-1$ for all $i$, and $a_{i} \geq 1$ if $i>n$. 
Genus 4. Now consider a 1 -form $(X, \omega) \in \Omega \mathcal{M}_{4}\left(2^{3}, 0^{3}\right)^{-}$, with associated involution $J$. Since $|\operatorname{Fix}(J)|=6=|\chi(X)|$, the quotient Riemann surface $A=X / J$ has genus one. Moreover, the form $\omega^{2}$ is $J$-invariant, so it descends to a meromorphic quadratic differential $q$ on $X$, with 3 simple poles and 3 simple zeros. Marking the poles by $P$, we obtain a form $(A, P, q) \in Q \mathcal{M}_{1,3}\left(-1^{3}, 1^{3}\right)$. Conversely, given a quadratic differential in this stratum, passage to the Riemann surface $X$ where $\omega=\sqrt{q}$ becomes single-valued defines a point $(X, \omega) \in \Omega \mathcal{M}_{4}\left(2^{3}, 0^{3}\right)^{-}$.

Summing up, we have a natural algebraic isomorphism

$$
\mathrm{sq}: \Omega \mathcal{M}_{4}\left(2^{3}, 0^{3}\right)^{-} \cong Q \mathcal{M}_{1,3}\left(-1^{3}, 1^{3}\right),
$$

respecting the action of $\mathrm{SL}_{2}(\mathbb{R})$.

Proof of Theorem 4.1. Let $(X, \omega)$ be a form in $\Omega \mathcal{M}_{4}\left(2^{3}, 0^{3}\right)^{-}$, with

$$
\operatorname{sq}(X, \omega)=(A, P, q) .
$$

Let $J$ be the unique involution such that $J^{*}(\omega)=-\omega$, and let $\operatorname{Fix}(J)=$ $Z^{\prime} \cup P^{\prime}$ where $\left|Z^{\prime}\right|=\left|P^{\prime}\right|=3$ and $Z^{\prime}=Z(\omega)$. We have a natural degree two map $\phi: X \rightarrow A$, injective on $\operatorname{Fix}(J)$, such that

$$
(q)=Z-P=\phi\left(Z^{\prime}\right)-\phi\left(P^{\prime}\right) .
$$

We will show that $(X, \omega) \in \Omega G \Longleftrightarrow(A, P, q) \in Q F$.

From gothic to flex. First assume that $(X, \omega) \in \Omega G$. We then have a degree three map to an elliptic curve, $p: X \rightarrow B$, and an involution $j \in \operatorname{Aut}(B)$, such that

$$
p(J(x))=j(p(x)) .
$$

By the definition of $\Omega G, Z^{\prime}$ is a fiber of $p$.

Choose the origin in $B$ so that $j(x)=-x$, and let $r: B \rightarrow B / j \cong \mathbb{P}^{1}$ be the quotient map. Then $r \circ p: X \rightarrow \mathbb{P}^{1}$ is a $J$-invariant map of degree 6. Consequently we have a unique degree three rational map $\pi: A \rightarrow \mathbb{P}^{1}$ making the diagram:

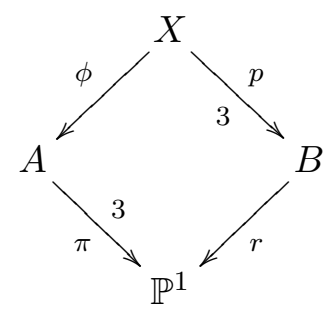

commute. We will show that: 
(i) the fibers of $\pi$ are linearly equivalent to $P$, and

(ii) $P$ is contained in the cocritical points of $\pi$.

To see (i), simply note that $Z$ is a fiber of $\pi$ since $Z^{\prime}$ is a fiber of $r \circ p$, and equation (4.2) shows that $Z$ is linearly equivalent to $P$ because the canonical bundle of $A$ is trivial.

To prove (ii), note that $p$ maps $\operatorname{Fix}(J)$ into $\operatorname{Fix}(j)$ by (4.3), and that $\operatorname{Fix}(j)=B[2]$ coincides with the set of 2 -torsion points in $B$. Let us denote the points of $B[2]$ by $\left\{e_{1}^{\prime}, e_{2}^{\prime}, e_{3}^{\prime}, e_{4}^{\prime}\right\}$, with $p\left(Z^{\prime}\right)=\left(e_{4}^{\prime}\right)$. Since $p^{-1}\left(e_{i}^{\prime}\right)$ is also $J$-invariant for $i=1,2,3$, we can find $P_{i}^{\prime}, Q_{i}^{\prime} \in X$ such that, as divisors, we have

$$
p^{-1}\left(e_{i}^{\prime}\right)=P_{i}^{\prime}+Q_{i}^{\prime}+J\left(Q_{i}^{\prime}\right)
$$

and $P_{i}=\phi\left(P_{i}^{\prime}\right) \in P$. Let $Q_{i}=\phi\left(Q_{i}^{\prime}\right)$ and let $e_{i}=r\left(e_{i}^{\prime}\right)$. Then again as divisors, we have

$$
\pi^{-1}\left(e_{i}\right)=P_{i}+2 Q_{i}
$$

for $i=1,2,3$, and hence $P_{i}$ is a cocritical point of $\pi$. This proves (ii) and shows that $(A, P, q) \in Q F$.

From flex to gothic. Now suppose $(A, P, q) \in Q F\left(-1^{3}, 1^{3}\right)$. Let $\pi: A \rightarrow$ $\mathbb{P}^{1}$ be a rational map of degree three verifying conditions (i) and (ii) above. Let $P=\left\{P_{1}, P_{2}, P_{3}\right\}$, let $\pi\left(P_{i}\right)=e_{i}$ for $i=1,2,3$, and let $\pi(Z)=e_{4}$. Then we can find $Q_{i} \in A$ such that (4.5) holds. Let $Q=Q_{1}+Q_{2}+Q_{3}$.

Let $r: B \rightarrow \mathbb{P}^{1}$ be an elliptic curve, presented as a 2 -fold covering of $\mathbb{P}^{1}$ branched over $E=\left\{e_{1}, e_{2}, e_{3}, e_{4}\right\}$. One can also regard $B / \mathbb{P}^{1}$ as the Riemann surface of the function $\sqrt{f}$, where

$$
(f)=e_{1}+e_{2}+e_{3}-3 e_{4} .
$$

Now note that, because the divisors $Z, P$ and $Q$ are all linearly equivalent,

$$
D=(f \circ \pi)-(q)=(P+2 Q-3 Z)-(Z-P)=2 Q+2 P-4 Z
$$

is the divisor of the square of a rational function on $A$. Thus $\sqrt{q}$ and $\sqrt{f \circ \pi}$ define the same Riemann surface $X / A$, and hence the map $\pi: A \rightarrow \mathbb{P}^{1}$ lifts to a map $p: X \rightarrow B$ making diagram (4.4) commute. This lift intertwines the $\mathbb{Z} / 2$ Galois groups of $X / A$ and $B / \mathbb{P}^{1}$, so $p$ is odd. Then, since $Z^{\prime}$ is a fiber of $p$, the form $(X, \omega)$ belongs to $\Omega G$. 


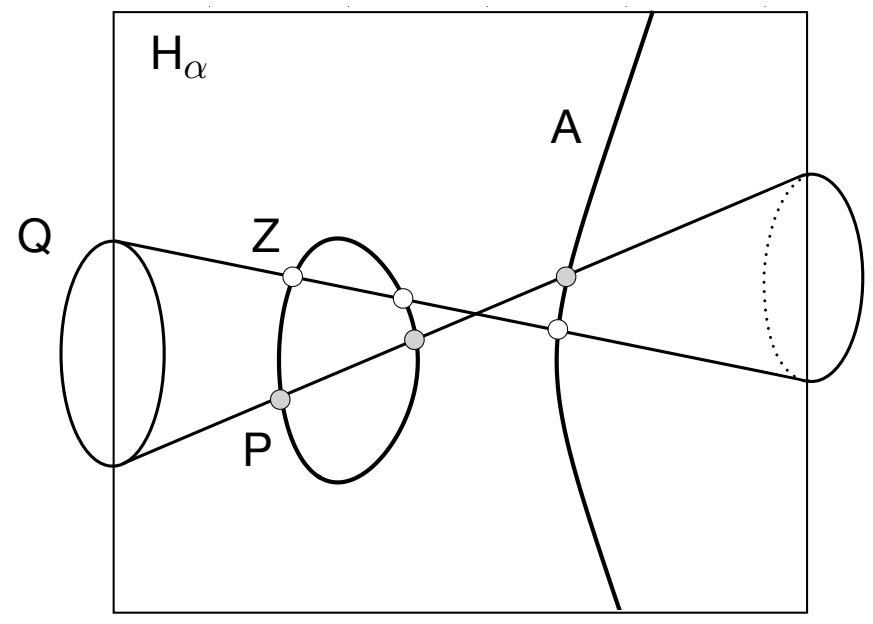

Figure 3. The canonical model for $X \subset \mathbb{P}^{3}$ gives six distinguished points $Z \cup P$ on the cubic curve $A$.

Remarks on the canonical model. The canonical embedding gives an illuminating geometric picture of the relationship between $X$ and $A$.

Let $(X, \omega)$ be a 1 -form in $\Omega \mathcal{M}_{4}\left(2^{3}, 0^{3}\right)^{-}$with involution $J$ and quotient curve $A=X / J$ of genus one. The eigenspaces of $J$ determine a splitting

$$
\Omega(X)=\Omega(A) \oplus \Omega(A)^{\perp},
$$

where we have identified $\Omega(A)=\mathbb{C} \alpha$ with the span of a $J$-invariant form $\alpha \in \Omega(X)$.

Let $\operatorname{Fix}(J)=Z \cup P$, where $Z=Z(\omega)$. We have $|Z|=|P|=3$. An involution of a hyperelliptic curve has at most 4 fixed points, provided it is not the hyperelliptic involution itself. Since $|\operatorname{Fix}(J)|=6$, the curve $X$ is not hyperelliptic. Thus its canonical linear system provides an embedding

$$
X \subset \mathbb{P} \Omega(X)^{*} \cong \mathbb{P}^{3} .
$$

Every nonzero 1-form $\eta$ on $X$ determines a plane $H_{\eta} \subset \mathbb{P}^{3}$.

It is classical that the space curve $X$, of degree six, is the transverse intersection $Q \cap C$ of an irreducible quadric and a cubic surface in $\mathbb{P}^{3}[\mathrm{GH}$, p.258]. The surface $Q$ is uniquely determined by $X$, but $C$ is not.

The automorphism $J$ acts naturally on $\Omega(X)$, and hence on $\mathbb{P}^{3}$. Its fixed-point set in $\mathbb{P}^{3}$ is the union of the plane $H_{\alpha}$ and the point $H_{\alpha}^{\perp}$ dual to 
$\Omega(A)^{\perp}$. Projection from this point yields a $J$-invariant map

$$
\Phi: X \rightarrow H_{\alpha} .
$$

The fibers of $\Phi$ consist of pairs of points that are interchanged by $J$. Hence $A=\Phi(X) \subset H_{\alpha}$ is a cubic plane curve, naturally isomorphic to $X / J$; and the fixed-points of $J \mid X$ are given simply by

$$
\operatorname{Fix}(J)=Z \cup P=X \cap H_{\alpha}=A \cap Q .
$$

We can now make the cubic surface defining $X$ canonical, by letting $C$ be the cone over $A$ with vertex $H_{\alpha}^{\perp}$.

The conic $Q \cap H_{\alpha}$ is singular, since its intersection with the line $H_{\omega} \cap H_{\alpha}$ consists of the three points $Z$. Since $J(Q)=Q$, this implies that $Q$ itself is singular. Thus $Q \cap H_{\alpha}$ is a pair of lines through the singular point of $Q-$ one of the form $H_{\omega} \cap H_{\alpha}$, containing $Z=Z(\omega)$ - and the other containing the remaining fixed-points $P$. See Figure 3.

We remark that the fact that $Q$ is singular implies:

$X$ lies on the $\theta$-null divisor $\mathcal{M}_{4}^{\prime} \subset \mathcal{M}_{4}$.

See [Te], [Gen, Theorem 1.3] and [ACGH, Ex. A-3, p.196] for more details.

Equations for $\boldsymbol{X} \in \boldsymbol{G}$. Using equations (2.10) and (2.11), we can now give an explicit formula for the canonical model of any $X \in G$. Namely, we find that $X=C \cap Q$ can be defined in affine coordinates by the two equations:

$$
y^{3}+b(x) y^{2}+c(x)=0 \text { and } z^{2}=(y+b(x))(x+a) .
$$

Here $a \in \mathbb{C}, \operatorname{deg}(b) \leq 1, \operatorname{deg}(c) \leq 3$, and $H_{\omega}=Z(x+a)$. Moreover, the associated elliptic curve $B$ is defined by

$$
t^{2}=-c(x)(x+a)
$$

and the degree three map $p: X \rightarrow B$ is given by the substitution $t=y z$.

One can also use equation (4.7) to show directly that $\operatorname{dim} G \geq 3$. Namely, if we fix $c(x)=x^{3}-1$ and vary $a$ and $b$, then equation (4.7) determines a rational map $\Phi: \mathbb{C}^{3} \rightarrow G$; and one can verify that $\Phi$ is finite-to-one by considering the seven critical values of $f: X \rightarrow B \rightarrow \mathbb{P}^{1}$.

Linear systems and rational maps. We conclude by describing some of the previously considered maps and spaces more intrinsically.

Let $(X, \omega)$ be a form in $\Omega G$, with associated genus one quotients $A$ and $B$. Let $(A, P, q)$ be the corresponding point in $Q F$, with associated map $\pi: A \rightarrow \mathbb{P}^{1}$. Let $\left(\alpha, \beta, \omega, \omega^{\prime}\right)$ be an orthogonal basis for $\Omega(X)$, where $\alpha$ and $\beta$ are pulled back from $A$ and $B$ respectively. Then: 
1. The degree six map $f: X \rightarrow \mathbb{P}^{1}$, factoring through $A$ or $B$ as in the commutative diagram (4.4), can be presented in the form $f=\omega / \omega^{\prime}$;

2. A basis for $Q F(A, P, \pi)$ is given by the $J$-invariant quadratic differentials $q=\omega^{2}$ and $q^{\prime}=\omega^{\prime} \omega$;

3. The degree three map $\pi: A \rightarrow \mathbb{P}^{1}$ can be expressed as $\pi=q^{\prime} / q$; and

4. The tangent space to the sheet of $F$ defined by $\pi$ is the kernel of the cotangent vector $\beta \omega \in Q(A, P)$.

To see (1), note that the fibers of $f$ as originally defined are divisors of canonical forms that push forward to 0 on $A$ and $B$. Points (2) and (3) follow easily. For (4), to show e.g. that

$$
\int_{A} \beta \omega \frac{\bar{q}^{\prime}}{\left|\bar{q}^{\prime}\right|}=\frac{1}{2} \int_{X} \beta \bar{\omega}^{\prime}|f|=0,
$$

observe that $\beta|f|$ is pulled back from $B$, while the pushforward of $\omega^{\prime}$ to $B$ is zero.

Note that $(\beta \omega)=W-P$ only depends on $\pi$ : the zeros of $\beta \omega$ are the three critical points of $\pi$ satisfying $\pi(W) \cap \pi(P)=\emptyset$.

\section{$5 \quad F$ is totally geodesic}

In this section we will prove our main results:

I. The locus $\Omega G$ is locally defined by real linear equations in period coordinates;

II. The loci $\Omega G$ and $Q F$ are $\mathrm{SL}_{2}(\mathbb{R})$-invariant; and

III. The flex locus $F \subset \mathcal{M}_{1,3}$ is a totally geodesic surface.

These assertions correspond to Theorems 1.1 and 1.4 and Corollary 1.5.

Here and in the sequel, cohomology is taken with complex coefficients unless otherwise specified.

Invariant varieties and period coordinates. We first remark that (I) implies (II) by general principles.

Recall that for any point $\left(X_{0}, \omega_{0}\right) \in \Omega \mathcal{M}_{g}$, there exists a neighborhood $U$ of $\left(X_{0}, \omega_{0}\right)$ (or an orbifold chart) in its stratum and a natural open analytic embedding

$$
\iota: U \hookrightarrow H^{1}\left(X_{0}, Z\left(\omega_{0}\right)\right) \cong \mathbb{C}^{2 g+\left|Z\left(\omega_{0}\right)\right|-1},
$$


called the period map, that sends $(X, \omega)$ to the unique relative cohomology class $[\omega]$ such that $\langle[\omega], \gamma\rangle=\int_{\gamma} \omega$. Using this map, we implicitly identify $U$ with an open subset of the vector space on the right.

A closed subset $M \subset \Omega \mathcal{M}_{g}\left(a_{1}, \ldots, a_{n}\right)$ is locally defined by real linear equations if, for each point $\left(X_{0} \omega_{0}\right) \in M$ and $U$ as above, there exists a finite set of complex subspaces $S_{i} \subset H^{1}\left(X_{0}, Z\left(\omega_{0}\right)\right)$, each invariant under $v \mapsto \bar{v}$, such that

$$
U \cap M=U \cap\left(S_{1} \cup \cdots \cup S_{k}\right) .
$$

The conclusion of $(I)$ means that $\Omega G$ has this form.

There is a natural action of the connected group $\mathrm{GL}_{2}^{+}(\mathbb{R})$ on any complex vector space $V$ with a real structure, defined by

$$
\left(\begin{array}{ll}
a & b \\
c & d
\end{array}\right) \cdot v=\left(\begin{array}{ll}
1 & i
\end{array}\right)\left(\begin{array}{ll}
a & b \\
c & d
\end{array}\right)\left(\begin{array}{l}
\operatorname{Re} v \\
\operatorname{Im} v
\end{array}\right) .
$$

The action of $\mathrm{SL}_{2}(\mathbb{R})$ on $\Omega \mathcal{M}_{g}$ has this form in period coordinates (see e.g. $[\mathrm{Mc} 1, \S 3])$. Evidentally any subspace of $V$ defined over $\mathbb{R}$ is $\mathrm{SL}_{2}(\mathbb{R})$ invariant, and so:

$$
\begin{aligned}
& \text { Any closed set } M \subset \Omega \mathcal{M}_{g} \text { locally defined by } \\
& \text { real linear equations is } \mathrm{SL}_{2}(\mathbb{R}) \text { invariant. }
\end{aligned}
$$

(The assumption that $M$ is closed is used to prove the group action is global.) Thus (I) implies (II) for $\Omega G$, and invariance is inherited by $Q F$ via Theorem 4.1, using the fact that the squaring map in equation (4.1) respects the action of $\mathrm{SL}_{2}(\mathbb{R})$.

A criterion for linearity. For the proof of (I) we will use the following general result.

Theorem 5.1 Let $M \subset \Omega \mathcal{M}_{g}\left(a_{1}, \ldots, a_{n}\right)$ be an algebraic variety whose irreducible components have dimension $\geq d$. Suppose that for every $(X, \omega) \in$ $M$ there is a d-dimensional subspace $S$, defined over a real number field, such that

$$
[\omega] \in S \subset H^{1}(X, Z(\omega)) .
$$

Then $M$ is locally defined by real linear equations in period coordinates, and $\operatorname{dim}(M)=d$.

Proof. Fix a point $(X, \omega) \in M$. Let $\mathcal{S}$ be the countable collection of all $d-$ dimensional subspaces $S$ as above. Then there exists a ball $U$ about $(X, \omega)$ 
in period coordinates such that $M \cap U$ is an analytic variety of dimension $\geq d$, contained in $\bigcup \mathcal{S}$. Consequently $\operatorname{dim}(M \cap U)=d$.

By Noetherian properties of analytic sets, $S \cap M \cap U$ has nonempty interior in $S$ for only finitely many subspaces $\left\{S_{1}, \ldots, S_{n}\right\} \subset \mathcal{S}$. Since $S_{i} \cap U$ is connected, it is contained in $M$ for all $i$. By the Baire category theorem, $\bigcup_{1}^{n}\left(S_{i} \cap U\right)$ is dense in $M \cap U$. Since $\bigcup_{1}^{n} S_{i}$ is also closed, this implies that $M \cap U=\bigcup_{1}^{n} S_{i} \cap U$.

The Abelian surface $\boldsymbol{C}(\boldsymbol{X} \mid \boldsymbol{A}, \boldsymbol{B})$. Recall from equation (1.2) that any $(X, \omega) \in \Omega G$, with associated maps $X \rightarrow A$ and $X \rightarrow B$ of degrees 2 and 3 respectively, determines a polarized Abelian surface

$$
C=C(X \mid A, B)=\operatorname{Jac}(X) / \operatorname{Im}(\operatorname{Jac}(A) \times \operatorname{Jac}(B)) .
$$

The submersion $\operatorname{Jac}(X) \rightarrow C$ determines an orthogonal splitting,

$$
\Omega(X) \cong \Omega(A) \oplus \Omega(B) \oplus \Omega(C),
$$

with $\omega \in \Omega(C)$. Similarly, on the level of relative cohomology, we obtain an exact sequence

$$
H^{0}(Z(\omega)) \rightarrow H^{1}(X, Z(\omega)) \rightarrow H^{1}(A) \oplus H^{1}(B) \oplus H^{1}(C) \rightarrow 0 .
$$

Since $J \mid Z(\omega)=\operatorname{Id}$ and $H^{1}(X)^{J}=H^{1}(A)$, we have a natural isomorphism

$$
H^{1}(B) \oplus H^{1}(C) \cong \operatorname{Ker}(J+\mathrm{Id}) \subset H^{1}(X, Z(\omega))
$$

defined over $\mathbb{Q}$.

The class $[\omega]$ lies in the $H^{1}(C)$ factor above, since $J^{*} \omega=-\omega$ and $\omega$ pushes forward to zero on $B$.

Proof of (I). By Theorems 3.3 and 4.1, $\Omega G$ is an irreducible 4-dimensional algebraic subvariety of $\Omega \mathcal{M}_{4}(2,2,2)$. As we have just seen, the relative periods of any form $(X, \omega) \in \Omega G$ lie in a 4-dimensional subspace $H^{1}(C) \subset$ $H^{1}(X, Z(\omega))$ defined over $\mathbb{Q}$. By Theorem 5.1 , these two facts imply that $\Omega G$ is locally defined by real linear equations in period coordinates.

Proof of (III). Recall that the Teichmüller norm on the cotangent space $Q(A, P)$ is given by $\|q\|=\int_{X}|q|$, and that the Beltrami coefficient $\bar{q} /|q|$ represents a unit vector tangent to the Teichmüller geodesic generated by $q$.

By Theorem $3.2, F$ is an immersed smooth surface. Let $(A, P)$ be a point on a sheet of $F$ with associated degree three map $\pi: A \rightarrow \mathbb{P}^{1}$. Let $T_{1}$ 
be the unit tangent space to this sheet at $(A, P)$, and let $Q_{1}$ be the unit ball in $Q F(A, P, \pi)$. Note that $T_{1}$ and $Q_{1}$ are both homeomorphic to $S^{3}$.

Consider the continuous map $\mu: Q_{1} \rightarrow T_{1}$ given by

$$
\mu: q \mapsto \frac{\bar{q}}{|q|}
$$

By uniqueness of the Teichmüller mapping, $\mu$ is injective, so by invariance of domain, it is a homeomorphism. Thus every complex geodesic tangent to $F$ at $(A, P)$ is contained in $F$, because it is the projection of an $\mathrm{SL}_{2}(\mathbb{R})$-orbit in $Q F$.

Defining equations for $\boldsymbol{\Omega} \boldsymbol{G}$. The proof of (I) also yields the following more precise result:

Theorem 5.2 Given $(X, \omega) \in \Omega G$ with associated Abelian surface $C=$ $C(X \mid A, B)$, there exists a neighborhood $U$ of $[\omega]$ in period coordinates such that

$$
[\omega] \in U \cap H^{1}(C) \subset \Omega G .
$$

Proof. Use the bundle $Q \widetilde{F} \rightarrow \widetilde{F}$ from the proof of Theorem 3.3, along with Theorem 4.1, to construct a 4-dimensional sheet of $\Omega G$ through $(X, \omega)$ along which $H^{1}(C)$ is constant, and use local injectivity of the period map.

\section{Remarks on linear and totally geodesic varieties.}

1. Statement (5.1) has a converse [Mo2, Prop. 1.2]: any $\mathrm{GL}_{2}^{+}(\mathbb{R})$-invariant analytic subvariety $V \subset \Omega \mathcal{M}_{g}$ is locally defined by real linear equations. Remarkably, the closure of any $\mathrm{GL}_{2}^{+}(\mathbb{R})$ orbit is also locally linear $[\mathrm{EMM}]$.

2. In contrast to the case of Teichmüller curves, whenever $V \subset \mathcal{M}_{g, n}$ is a totally geodesic variety of dimension two or more, a typical complex geodesic in $V$ is generated by a truly quadratic differential $q$. That is, $q$ is not the square of a 1 -form. Thus it is useful to work directly with $Q \mathcal{M}_{g, n}$ when studying such $V$.

3. There are also interesting higher-dimensional $\mathrm{SL}_{2}(\mathbb{R})$-invariant varieties $M \subset \Omega \mathcal{M}_{g}$ which, unlike $\Omega G$, are unrelated to totally geodesic varieties. The prime examples are the eigenform loci in genus $g=2,3$ and 4 [Mc3].

4. It is known that the only complex symmetric space that can occur as an immersed totally geodesic submanifold of $\mathcal{T}_{g, n}$ is the unit disk [An]. 


\section{$6 \quad F$ is primitive}

A holomorphic 1-form $(X, \omega) \in \Omega \mathcal{M}_{g}$ is primitive unless there is a form $(Y, \eta) \in \Omega \mathcal{M}_{h}, h<g$, and a holomorphic map $p: X \rightarrow Y$ such that $\omega=p^{*}(\eta)$. A similar definition applies to quadratic differentials.

In this section we will show:

I. A typical form in $Q F$ or $\Omega G$ is primitive; and therefore:

II. The surface $F$ does not arise via a covering construction.

Another proof of primitivity appears in the next section.

Covering constructions. Let $\left(\Sigma_{g}, \Pi_{n}\right)$ denote a smooth, oriented topological surface of genus $g$, with a set of $n$ marked points $\Pi_{n} \subset \Sigma_{g}$. Consider a branched covering map

$$
\pi:\left(\Sigma_{g}, \Pi_{n}\right) \rightarrow\left(\Sigma_{h}, \Pi_{m}\right)
$$

whose critical points $C(\pi)$ satisfy

$$
C(\pi) \cup \Pi_{n}=\pi^{-1}\left(\Pi_{m}\right) .
$$

Condition (6.1) can be weakened to $C(\pi) \subset \pi^{-1}\left(\Pi_{m}\right)$ if $(h, m)=(1,1)$. By pulling back complex structures, we then have an induced map

$$
F_{\pi}: \mathcal{T}_{h, m} \rightarrow \mathcal{M}_{g, n}
$$

Let $\mathcal{M}_{h, m}^{\prime}=\mathcal{T}_{h, m} / \Gamma$ be the quotient of Teichmüller space by the subgroup of finite index in $\operatorname{Mod}_{h, m}$ consisting of mapping classes that lift to $\left(\Sigma_{g}, \Pi_{n}\right)$. Then $F_{\pi}$ descends to give a holomorphic map

$$
f_{\pi}: \mathcal{M}_{h, m}^{\prime} \rightarrow \mathcal{M}_{g, n}
$$

Condition (6.1) insures that the lift of a Teichmüller mapping remains a Teichmüller mapping, and thus $f_{\pi}$ is a local isometry.

Let $V$ be a proper subvariety of $\mathcal{M}_{g, n}$ that is totally geodesic. We say $V$ arises via a covering construction if there exists a map $\pi$ as above, with $\operatorname{dim} \mathcal{M}_{h, m}<\operatorname{dim} \mathcal{M}_{g, n}$, and a totally geodesic variety $V_{0} \subset \mathcal{M}_{h, m}^{\prime}$, such that

$$
V=f_{\pi}\left(V_{0}\right) \subset \mathcal{M}_{g, n}
$$

(We allow $V_{0}=\mathcal{M}_{h, m}^{\prime}$ )

We say $V$ is primitive if it does not arise via a covering construction. 
Example. Given integers $a, b>0$, consider the surface $S_{a b} \subset \mathcal{M}_{1,3}$ defined by

$$
S_{a b}=\left\{(A, P): \begin{array}{l}
\text { for some ordering of the points of } P \\
\text { we have }\left[a P_{1}+b P_{2}\right]=\left[(a+b) P_{3}\right] \in \operatorname{Pic}(A)
\end{array}\right\} .
$$

We claim that:

$S_{11} \subset \mathcal{M}_{1,3}$ is a totally geodesic surface defined by a covering construction.

In fact, every $(A, P) \in S_{11}$ admits an involution swapping $P_{1}$ and $P_{2}$. The degree two quotient map $\pi:(A, P) \rightarrow\left(\mathbb{P}^{1}, Q\right)$ gives a sphere with 5 marked points - the critical values of $\pi$, and the common image of $P_{1}$ and $P_{2}$. Applying the covering construction, we obtain a totally geodesic immersion

$$
\mathcal{M}_{0,5}^{\prime} \rightarrow \mathcal{M}_{1,3}
$$

with image $S_{11}$.

Proof of (I) for $\boldsymbol{Q F}$. Since $\operatorname{dim} Q \mathcal{M}_{0,4}=2<\operatorname{dim} Q F=4$, the following statement makes precise the fact that most forms in $Q F$ are primitive.

Lemma 6.1 Every form in $Q \mathcal{M}_{1,3}\left(-1^{3}, 1^{3}\right)$ is either primitive or the pullback of a form in $Q \mathcal{M}_{0,4}$ via a covering construction.

Proof. Let $(A, P, q)$ be a form in $Q \mathcal{M}_{1,3}\left(-1^{3}, 1^{3}\right)$. Suppose $q=p^{*}\left(q^{\prime}\right)$ with $q^{\prime} \in Q\left(A^{\prime}, P^{\prime}\right)$ and $\operatorname{deg}(p)>1$. If $A^{\prime}$ has genus one, then $p$ must be a covering map of degree three; but then $q^{\prime}$ has just one pole, which is impossible. Thus $A^{\prime} \cong \mathbb{P}^{1}$. Any zero of $q^{\prime}$ must be simple, and have $\operatorname{deg}(p)$ simple pre-images; thus in the presence of a zero, we must have $\operatorname{deg}(p) \leq 3$ and $q^{\prime}$ must have at least 5 poles. This contradicts the fact that $q$ only has 3 poles. Thus $q^{\prime}$ has 4 poles and no zeros, i.e. $q^{\prime} \in Q \mathcal{M}_{0,4}$; and since $q$ only has simple zeros, $p$ can only be branched over the poles of $q^{\prime}$.

Proof of (II). Suppose $F \subset \mathcal{M}_{1,3}$ arises via a covering construction $f_{\pi}$ : $\mathcal{M}_{h, m}^{\prime} \rightarrow \mathcal{M}_{1,3}$. Then every complex geodesic contained in $F$ is generated by the lift of a quadratic differential in $Q \mathcal{M}_{h, m}$. Thus $Q F$ contains no primitive form, contrary to statement (I) above. 
Proof of (I) for $\boldsymbol{\Omega} \boldsymbol{G}$. For later applications to Teichmüller curves, we conclude by proving a similar primitivity theorem for forms in $\Omega G$.

Lemma 6.2 Every form in $\Omega \mathcal{M}_{4}\left(2^{3}, 0^{3}\right)^{-}$is either primitive or the pullback of a form in $\Omega \mathcal{M}_{1}$.

Proof. Let $(X, \omega)$ be a form in $\Omega \mathcal{M}_{4}\left(2^{3}, 0^{3}\right)^{-}$. Suppose $\omega=p^{*}(\eta)$, with $(Y, \eta) \in \Omega \mathcal{M}_{g}, 1<g<4$. By the Riemann-Hurwitz formula, we must have $g=2$ and $\operatorname{deg}(p)=2$ or 3 . But if $\operatorname{deg}(p)=2$ then $p$ has two simple critical points, contradicting the fact that $\omega$ has no simple zeros. Thus $p$ is a covering map of degree three, and $\eta$ has a single zero of multiplicity two.

Let $J$ be the unique involution of $X$ fixing $Z(\omega)$, and let $j$ be the hyperelliptic involution of $Y$. Since $J^{*}(\omega)=-\omega$, the map $p$ is odd; that is, $p(J(x))=j(p(x))$ for all $x \in X$. Thus $\left(X, \omega^{2}\right) / J$ is the pullback of the quadratic differential $\left(Y, \eta^{2}\right) / j$. Since the latter is not in $Q \mathcal{M}_{0,4}$, this contradicts Lemma 6.1.

\section{The Kobayashi metric on $F$}

In this section we give a more geometric proof that $F$ is primitive, by showing that $\mathcal{T} F$ is not isomorphic to any traditional Teichmüller space. The proof is a variation on a theme of Royden; it is based on an analysis of the Kobayashi metric.

The Kobayashi metric. Recall that $\mathcal{T} F$ denotes an irreducible component of the preimage of $F$ in $\mathcal{T}_{1,3}$. This smooth, contractible space is isomorphic, as a complex manifold, to the universal cover of the surface $\widetilde{F}$ introduced in $\S 3$. It is well-known that the Kobayashi and Teichmüller metrics agree on $\mathcal{T}_{g, n}[\mathrm{Ga}, \S 7]$. Similarly:

The Kobayashi and Teichmüller metrics agree on $\mathcal{T} F$, and the inclusion $\mathcal{T} F \subset \mathcal{T}_{1,3}$ is an isometry.

In fact, any two distinct points $x, y \in \mathcal{T} F$ lie on a unique complex geodesic $D \subset \mathcal{T}_{1,3}$; since $\mathcal{T} F$ is totally geodesic, we have $D \subset \mathcal{T} F \subset \mathcal{T}_{1,3}$; and since inclusions are contractions, $D$ is also a Kobayashi geodesic in $\mathcal{T} F$.

The vertices of a norm. Let $V$ be a 2-dimensional normed complex vector space. We say $V$ has $n$ vertices if the set of $v \in V$ where the function $\|v\|$ is real-analytic is the complement of $n$ distinct lines through the origin. To distinguish $\mathcal{T} F$ from $\mathcal{T}_{g, n}$, we will show: 
Theorem 7.1 The cotangent space at any point in $\mathcal{T}_{0,5}$ has 5 vertices in the Teichmüller norm, while the cotangent space at a typical point in $\mathcal{T} F$ has 6 .

Pairs of quadratic differentials. The following general result describes points where the Teichmüller norm is not smooth. Consider quadratic differentials $q_{0}, q_{1} \in Q(X, P)$, where $(X, P) \in \mathcal{M}_{g, n}$. Fix $x \in X$ and let $m_{i}=\operatorname{ord}_{x}\left(q_{i}\right)$ denote the order of vanishing of $q_{i}$ at $x$ (or -1 if $q_{i}$ has a simple pole there).

Lemma 7.2 If $m_{1}<\left(m_{0}-1\right) / 2$, then $f(t)=\left\|q_{0}+t q_{1}\right\|$ is not $C^{2}$ at $t=0$.

Proof. Note that $\left|q_{0}+t q_{1}\right|$ is a convex function of $t \in \mathbb{R}$, and if a sum of convex functions is $C^{2}$, then each function has a bounded (distributional) second derivative. Thus it suffices to show that $\int_{U}\left|q_{0}+t q_{1}\right|$ has an unbounded second derivative in some neighborhood $U$ of $x$; this is done in [Roy, §2] and, allowing simple poles, in [Ga, §9.4].

Proof of Theorem 7.1. First note that the function $\|q\|$ is real-analytic on any stratum of $Q \mathcal{M}_{g, n}$, since it can be expressed as a polynomial in the absolute periods of $\sqrt{q}$ and their complex conjugates. In particular, $\|q\|$ is real-analytic on the generic stratum $Q \mathcal{M}_{0,5}\left(-1^{5}, 1\right)$. Thus for any $(X, P) \in \mathcal{M}_{0,5}$, the norm is real-analytic except possibly on the 5 lines $V_{i} \subset Q(X, P)$ where $q=q_{0}$ has only 4 poles. For any such $q_{0}$, we can find an $x \in P$ and a $q_{1} \in Q(X, P)$ such that $q_{1}$ has a simple pole at $x$, but $q_{0}$ does not. Applying Lemma 7.2 with $\left(m_{0}, m_{1}\right)=(0,-1)$, we find that $\|q\|$ is not $C^{2}$ at $q_{0}$ and hence $Q(X, P)$ has 5 vertices.

Now consider the cotangent space $Q F(A, P, \pi)$ to a point $(A, P) \in \mathcal{T} F$. Note that for $S \in H A$, the projection $\pi_{S}: A \rightarrow \mathbb{P}^{1}$ has six simple critical values except in the finitely many cases where $S$ lies on the tangent line to a flex of $A$. Thus $\pi$ also has six critical values for a typical point in $\mathcal{T} F$.

Since $\mathcal{T} F$ is totally geodesic, the Teichmüller norm on its cotangent space at $(A, P)$ is the restriction of the Teichmüller norm on $Q(A, P)$. Recall that for any $q \neq 0$ in $Q(A, P, \pi)$, we have $(q)=Z-P$ where $Z$ is a fiber of $\pi$, and that the generic stratum here is $Q \mathcal{M}_{1,3}\left(-1^{3}, 1^{3}\right)$. Thus the norm is real-analytic except possibly along the 6 lines $V_{i} \subset Q F(A, P, \pi)$ where $\left(q_{0}\right)=Z-P$ and the support of $Z$ contains a simple critical point $x$ of $\pi$. In this case $x \notin P$, and hence $m_{0}=\operatorname{ord}_{x}\left(q_{0}\right)=2$, while $m_{1}=\operatorname{ord}_{x}\left(q_{1}\right)=0$ for most other $q_{1} \in Q F(A, P, \pi)$; thus $\|q\|$ fails to be $C^{2}$ at $q_{0}$ by Lemma 7.2. Therefore the normed cotangent space to $\mathcal{T} F$ typically has 6 vertices. 
Proof of Theorem 1.2. Suppose $\mathcal{T} F$ is isomorphic to a traditional Teichmüller space. Then we have $\mathcal{T} F \cong \mathcal{T}_{0,5} \cong \mathcal{T}_{1,2}$ as a complex manifold, since there is only one 2 -dimensional Teichmüller space up to isomorphism. Since the Kobayashi metric on a space depends only its complex structure, and agrees with the Teichmüller metric in these cases, this implies that the cotangent bundles of $\mathcal{T}_{0,5}$ and $\mathcal{T} F$ are isomorphic as bundles of normed vector spaces, contradicting Theorem 7.1.

Proof of Corollary 1.3. Since $F$ has codimension one, if it arises from a totally geodesic surface $V_{0} \subset \mathcal{M}_{h, m}^{\prime}$ via a covering construction then we must have $V_{0}=\mathcal{M}_{h, m}^{\prime}$ and hence $\mathcal{T} F \cong \mathcal{T}_{h, m}$, contrary to Theorem 1.2.

Remark. For a visualization of the Teichmüller norm on the cotangent space to $\mathcal{M}_{0,5}$, see $[\mathrm{Mu} 2]$.

\section{Teichmüller curves in $\mathcal{M}_{4}$}

A Teichmüller curve is geometrically primitive if it is generated by a primitive 1-form (in the sense of $\S 6$ ). Every Teichmüller curve arises from a unique geometrically primitive Teichmüller curve via a covering construction [Mo1, Theorem 2.5]. It is useful to work with 1-forms, rather than quadratic differentials, so the class $[\omega] \in H^{1,0}(X)$ can be discussed from the point of view of Hodge theory.

In this section we will show:

I. The locus $G_{D} \subset \mathcal{M}_{4}$ is a finite union of Teichmüller curves.

II. If $D$ is not a square, then every component of $G_{D}$ is geometrically primitive.

III. If the stabilizer of a form in $\Omega G$ contains a hyperbolic element $g$, then $\operatorname{SL}(X, \omega)$ is a lattice and $(X, \omega) \in \Omega G_{D}$ for some $D$ with $\mathbb{Q}(\operatorname{tr} g)=$ $\mathbb{Q}(\sqrt{D})$.

The first three assertions are restatements of Theorems 1.6, 1.7 and 1.8. Aside from (II), the proofs follow the same lines as the proofs in genus 2 given in [Mc1]. We remark that the curves $G_{D} \subset \mathcal{M}_{4}$ also descend to give isometrically immersed curves $F_{D} \subset F$.

Eigenforms. As in $\S 1$, given a discriminant $D>0$, we let $\Omega G_{D}$ denote the set of $(X, \omega) \in \Omega G$ with associated $A$ and $B$ such that: 
1. There exists a proper, self-adjoint action of $\mathcal{O}_{D}$ by endomorphisms of $C(X \mid A, B)$, and

2. $T^{*} \omega \in \mathbb{C} \omega$ for all $T \in \mathcal{O}_{D}$.

(Here proper means $\mathcal{O}_{D}$ is a maximal quadratic subring of $\operatorname{End}(C)$.) In brief, $(X, \omega)$ is an eigenform for real multiplication by $\mathcal{O}_{D}$. The projection of $\Omega G_{D}$ to $\mathcal{M}_{4}$ is denoted by $G_{D}$.

Proof of (I). We refine the discussion of $\S 5$. By the theory of Hilbert modular surfaces (cf. [vG]), the eigenforms for real multiplication by $\mathcal{O}_{D}$ form an algebraic subvariety of $\Omega G$ of codimension $\leq 2$. Thus every irreducible component of $\Omega G_{D}$ has dimension $\geq 2$. On the other hand, for any $(X, \omega) \in \Omega G_{D}$ we have an endomorphism $T \in \operatorname{End}(C)$ and a $\lambda \in K=\mathbb{Q}(\sqrt{D})$ such that $[\omega]$ belongs to the 2-dimensional subspace

$$
S=\operatorname{Ker}(T-\lambda I) \subset H^{1}(C) \subset H^{1}(X, Z(\omega)) .
$$

Applying Theorem 5.1 we find that $\Omega G_{D}$ is $\mathrm{SL}_{2}(\mathbb{R})$-invariant, and therefore $G_{D}$ is finite union of irreducible curves, each of which is totally geodesic.

Proof of (II). By Lemma 6.2, if $(X, \omega) \in \Omega G_{D}$ is not primitive then it is the pullback of a form of genus $h=1$. But then the $\mathcal{O}_{D}$-invariant subspace of $H^{1}(X, \mathbb{R})$ spanned by $[\operatorname{Re}(\omega)]$ and $[\operatorname{Im}(\omega)]$ is defined over $\mathbb{Q}$, so $D$ is a square.

Proof of (III). Let $\operatorname{Aff}(X, \omega)$ denote the group of affine automorphisms of $(X, \omega)$. The derivative $D \psi \in \mathrm{SL}_{2}(\mathbb{R})$ of any affine automorphism is constant in charts on $X-Z(\omega)$ where $\omega=d z$. The map $\psi \mapsto D \psi$ sends $\operatorname{Aff}(X, \omega)$ to $\operatorname{SL}(X, \omega)$, the stabilizer of $(X, \omega)$ in $\mathrm{SL}_{2}(\mathbb{R})$.

Suppose we have a hyperbolic element $g \in \operatorname{SL}(X, \omega)$. Replacing $g$ with $g^{2}$, we can assume that $\operatorname{tr}(g)>0$. Choose a element $\psi \in \operatorname{Aff}(X, \omega)$ with $D \psi=g$.

Let $C=C(X \mid A, B)$ as above. Given $X$, there are only finitely many choices for $A$ and $B$, and they are locally preserved along an $\mathrm{SL}_{2}(\mathbb{R})$-orbit, so after replacing $\psi$ with an iterate we can assume it stabilizes $H^{1}(C) \subset$ $H^{1}(X)$.

Since $\psi^{*} \mid H^{1}(C ; \mathbb{R})$ preserves a symplectic form, its eigenvalues come in reciprocal pairs, and its action is unitary for the Hermitian form $\langle\alpha, \beta\rangle=$ $(i / 2) \int_{X} \alpha \wedge \bar{\beta}$ on $H^{1}(C)$. Thus the map

$$
T=\psi^{*}+\left(\psi^{-1}\right)^{*} \mid H^{1}(C)
$$


is self-adjoint, its eigenspaces are orthogonal and their dimensions are even.

After replacing $(X, \omega)$ by another point in its $\mathrm{SL}_{2}(\mathbb{R})$ orbit, we can assume that

$$
D \psi=\left(\begin{array}{cc}
\lambda & 0 \\
0 & \lambda^{-1}
\end{array}\right) .
$$

Then $\psi$ is a pseudo-Anosov mapping, with invariant foliations defined by the harmonic forms $\operatorname{Re}(\omega)$ and $\operatorname{Im}(\omega)$. These forms give a $\psi^{*}$-eigenbasis for a 2-dimensional subspace $V \subset H^{1}(C)$, with eigenvalues $\lambda$ and $\lambda^{-1}$. By the theory of pseudo-Anosov mappings, these eigenvalues are simple and all other eigenvalues of $\psi^{*} \mid H^{1}(C)$ have modulus less than $\lambda$.

In particular, the eigenvalues of $T \mid H^{1}(C)$ are given by $t$ and $t^{\prime}$, where $t=\lambda+\lambda^{-1}$ and $\left|t^{\prime}\right|<t$; The corresponding eigenspaces $V$ and $V^{\prime}$ satisfying $V^{\prime}=V^{\perp}$.

Extend $\{\omega\}$ to an orthogonal basis $\left\{\omega, \omega^{\prime}\right\}$ for $\Omega(C)$. Then the cohomology classes $[\omega],\left[\omega^{\prime}\right]$ give a basis for $H^{1,0}(C)$ consisting of eigenvectors for $T$. Consequently $T$ preserves $H^{1,0}(C)$. Since $T$ also preserves $H^{1}(C, \mathbb{Q})$, by replacing $T$ with $n T$ for some $n>0$, we can insure that $T \in \operatorname{End}(C)$.

The ring $\mathbb{Z}[T]$ is quadratic since $T$ has exactly two eigenvalues. Let $D$ be the discriminant of the maximal quadratic order in $\operatorname{End}(C)$ containing $\mathbb{Z}[T]$. Then $(X, \omega) \in \Omega G_{D}$, and hence $\operatorname{SL}(X, \omega)$ is a lattice by (I). The field $K=\mathbb{Q}(\operatorname{tr} g)$ is the same as the field generated by the eigenvalues of $T$, so $K=\mathbb{Q}(\sqrt{D})$.

\section{Explicit polygonal constructions}

In this section we will prove Theorems 1.9 and 1.10. The latter gives new examples of Teichmüller curves in $\mathcal{M}_{4}$. In the course of the proof we will give explicit equations for $\Omega G$ in period coordinates.

Cylinder deformations. We begin with some notation. We write the usual action of $\gamma \in \mathrm{GL}_{2}^{+}(\mathbb{R})$ on $\Omega \mathcal{M}_{g}$ by

$$
(X, \omega) \mapsto \gamma \cdot(X, \omega) .
$$

Let $C \subset X$ be a collection of parallel cylinders, and let $\phi:(X, \omega) \rightarrow\left(X^{\prime}, \omega^{\prime}\right)$ be a PL map for the flat metrics $|\omega|$ and $\left|\omega^{\prime}\right|$. We say $\left(X^{\prime}, \omega^{\prime}\right)$ is a cylinder deformation of $(X, \omega)$, and write

$$
\left(X^{\prime}, \omega^{\prime}\right)=\gamma_{C} \cdot(X, \omega),
$$

if $D \phi=\gamma$ on $C$ and $D \phi=$ Id on the rest of $X$ (cf. [Wr3]). 
Note that we must have $\gamma(v)=v$ on vectors $v$ parallel to $\partial C$; in particular, if $\operatorname{det}(\gamma)=1$ then $\gamma$ is a shear. Conversely, any linear map $\gamma$ that fixes vectors parallel to $\partial C$ determines a new form $\gamma_{C} \cdot(X, \omega) \in \Omega \mathcal{M}_{g}$.
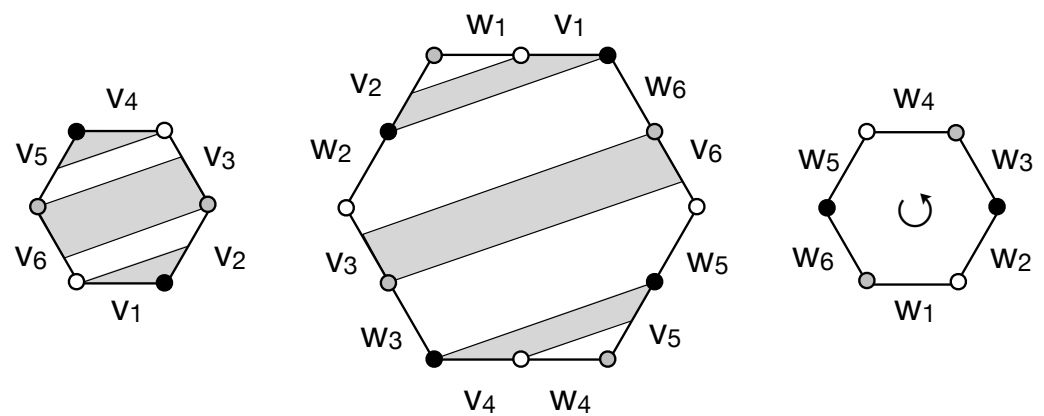

Figure 4. Hexagon form $\left(X_{0}, \omega_{0}\right)$ in $\Omega G$, and parallel cylinders $C \subset X_{0}$.

A symmetric form in $\Omega \boldsymbol{G}$. Now consider the holomorphic 1-form $\left(X_{0}, \omega_{0}\right) \in$ $\Omega \mathcal{M}_{4}(2,2,2)$ obtained by gluing together three regular hexagons along parallel edges as indicated in Figure 4. The three zeros of $\omega$ are indicated by white, black and gray dots. The edges are oriented to run counter-clockwise around the right and left hexagons, in the complex directions $v_{i}, w_{i} \in \mathbb{C}$. We claim:

The form $\left(X_{0}, \omega_{0}\right)$ lies in the gothic locus $\Omega G$.

In fact, rotation of each hexagon by $60^{\circ}$ preserves the gluing pattern, and thus descends to an automorphism $T: X_{0} \rightarrow X_{0}$ that cyclically permutes the three zeros of $\omega_{0}$. If we let $J=T^{3}$ and define an elliptic curve by $B=$ $X_{0} /\left\langle T^{2}\right\rangle$, then $J^{*} \omega_{0}=-\omega_{0}$ and the degree three quotient map $\pi: X_{0} \rightarrow B$ sends $Z\left(\omega_{0}\right)$ to a single point. Thus $\left(X_{0}, \omega_{0}\right)$ belongs to $\Omega G$ by the definition in $\S 1$.

Period coordinates. We can regard $v_{i}$ and $w_{i}$ as coordinates on $H^{1}\left(X_{0}, Z\left(\omega_{0}\right)\right)$, since each oriented edge connects two zeros of $\omega_{0}$.

In fact, these 12 vectors span $H_{1}\left(X_{0} ; Z\left(\omega_{0}\right)\right) \cong \mathbb{C}^{10}$, and are subject to the 2 relations $\sum v_{i}=\sum w_{i}=0$. For the picture at hand, we have $v_{i}\left(\omega_{0}\right)=w_{i}\left(\omega_{0}\right)=\zeta^{i-1}$, where $\zeta=\exp (\pi i / 3)$.

In these coordinates, a sheet of $\Omega G$ is locally defined by the linear relations:

$$
\begin{aligned}
& v_{i+3}=-v_{i} \text { and } w_{i+3}=-w_{i}, \quad i=1,2,3 ; \quad \text { and } \\
& v_{1}+v_{3}+v_{5}=w_{1}+w_{3}+w_{5}=0 .
\end{aligned}
$$


The first set of equations insures that $J^{*}(\omega)=\omega$, while the second insures that $\omega \in H^{1}(B)^{\perp}$. To see that the second condition is correct, just note that $v_{1}+v_{3}+v_{5}$ and $w_{1}+w_{3}+w_{5}$ span the $T^{2}$-invariant subspace of $H_{1}\left(X_{0}\right)$, and hence represent a basis for $H_{1}(B)$. These equations define an open subset of a sheet of $\Omega G$ by Theorem 5.2.

Cylinders and $\boldsymbol{H}_{\mathbf{1}}(\boldsymbol{B})$. The shaded region in Figure 4 covers a collection of three parallel cylinders, $C=C_{1} \cup C_{2} \cup C_{3} \subset X$. We claim:

Any cylinder deformation of the form $(X, \omega)=\gamma_{C} \cdot\left(X_{0}, \omega_{0}\right)$ also lies in $\Omega G$.

To see this, it suffices to show that shearing $C$ preserves the period conditions in (9.2). The first set is preserved because $J(C)=C$. As for the second set, by examining the left hexagon in Figure 4, we see that the relative cycle $v=2 v_{1}-\left(v_{1}+v_{2}+v_{6}\right)$ can be represented by two arcs lying entirely in $C$. But modulo the first set of equations, we have $v=v_{1}+v_{3}+v_{5}$; thus $v\left(\omega_{0}\right)=0$, and hence $v(\omega)=0$. Similarly, the cycle $w_{1}+w_{3}+w_{5}$ can be represented by arcs outside of $C$, so its vanishing is also preserved by $\gamma_{C}$.

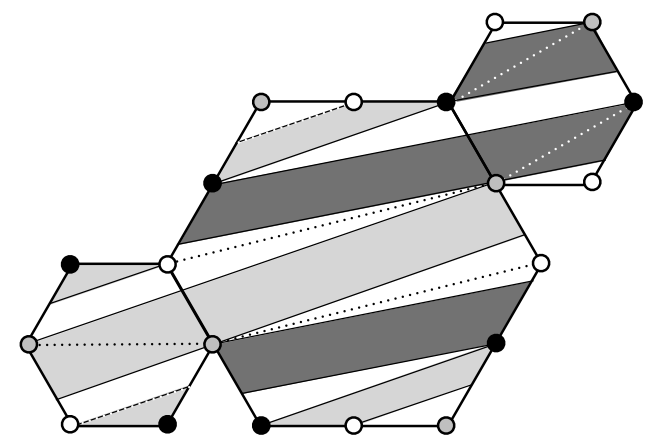

Figure 5. Preparation for cut and paste.

PL maps. Figure 5 gives another presentation of the surface $\left(X_{0}, \omega_{0}\right)$, in which the dark shaded regions cover two more parallel cylinders $D=D_{1} \cup$ $D_{2} \subset X_{0}$. Since $J(D)=D$, and the cycles $v_{1}+v_{3}+v_{5}$ and $w_{1}+w_{3}+w_{5}$ can be represented by arcs outside of $D$, deformations of the form $\gamma_{D}^{\prime} \cdot\left(X_{0}, \omega_{0}\right)$ also lie in $\Omega G$. In fact, by combining $\gamma_{C}, \gamma_{D}^{\prime}$ and the action of $\mathrm{GL}_{2}^{+}(\mathbb{R})$, we can conclude:

If there exists a PL map $\phi:\left(X_{0}, \omega_{0}\right) \rightarrow(X, \omega)$ such that $D \phi$ is constant on $C, D$ and their complement, then $(X, \omega) \in \Omega G$. 


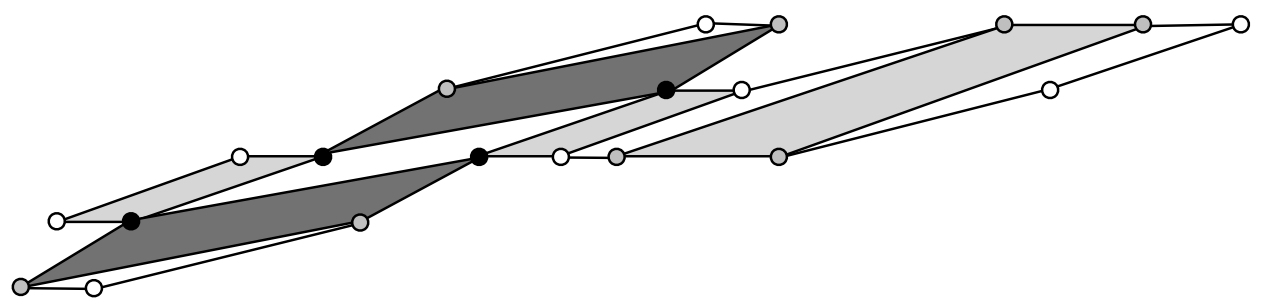

Figure 6. Result of cut and paste is a new polygon $P^{\prime}$.
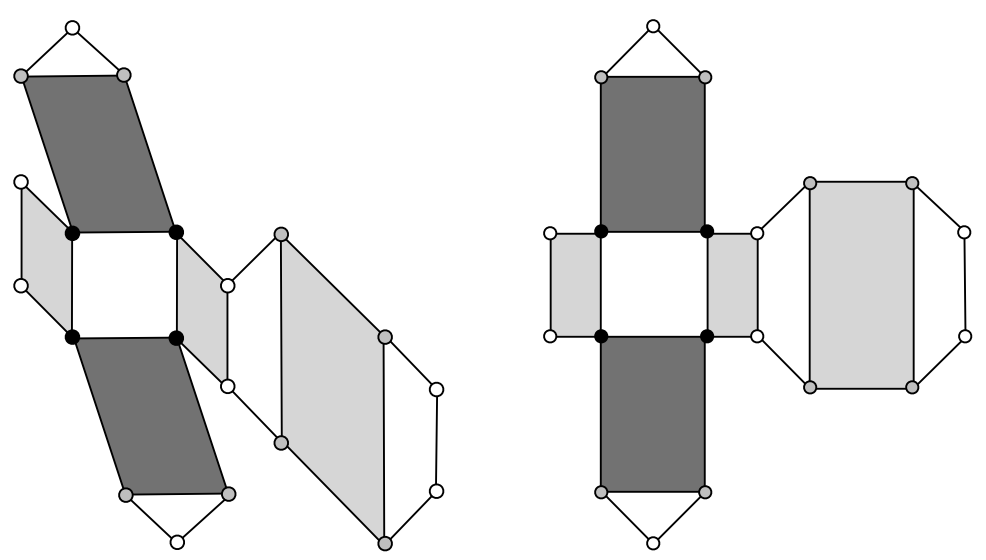

Figure 7. Linear image $\gamma\left(P^{\prime}\right)$, followed by shearing. 
With this information in hand, we may complete the:

Proof of Theorem 1.9. We first show that $(X, \omega)=P(a, b) / \sim$ is in $\Omega G$ for any $a, b>0$. Start with $\left(X_{0}, \omega_{0}\right) \in \Omega G$ as presented in Figure 5 . Cutting along the dotted lines and reassembling, we obtain a new polygon $P^{\prime}$ representing the same 1-form, shown in Figure 6. Every dotted edge is now part of the boundary of $P^{\prime}$.

Let $\gamma \in \mathrm{GL}_{+}(\mathbb{R})$ be the unique linear map that sends the pair of symmetric white triangles in Figure 6 to isosceles right triangles with horizontal bases of unit length. The result is a new 1-form $\left(X_{1}, \omega_{1}\right)=\gamma \cdot\left(X_{0}, \omega_{0}\right)$, which is the quotient of the polygon $\gamma\left(P^{\prime}\right)$ show in Figure 7 at the left. (In fact, if we allow complex values of $a, b$, then $\left.P^{\prime}=P((1-i) / 2,(3+i) / 2)\right)$.

Finally, by applying cylinder deformations, we can transform the light and dark regions into rectangles (as shown at the right) to produce the form $\left(X_{2}, \omega_{2}\right)=P(a, b) / \sim$ for any $a, b>0$. By observation (9.3), the resulting form is still in $\Omega G$.

Proof of Theorem 1.10. Now suppose $a, b \in K=\mathbb{Q}(\sqrt{d})$ satisfy equation (1.3). We may assume that $\sqrt{d}$ is irrational, since $(X, \omega)$ is squaretiled in the rational case. Note that the horizontal cylinders in $(X, \omega)$ have moduli $\left(m_{1}, m_{2}\right)=\left(b,(4 a+2)^{-1}\right)$, while the vertical cylinders have moduli $\left(m_{1}^{\prime}, m_{2}^{\prime}\right)=\left(a,(4 b+6)^{-1}\right)$. As is easily verified, equation (1.3) is exactly the condition needed to insure that $m_{1} / m_{2}$ and $m_{1}^{\prime} / m_{2}^{\prime}$ are rational.

This rationality implies that $\mathrm{Aff}^{+}(X, \omega)$ contains a vertical and a horizontal Dehn twist, whose product produces a hyperbolic element $\gamma \in \operatorname{SL}(X, \omega)$ (cf. $[\mathrm{Mc} 3, \S 4]$ ). The relative periods $\operatorname{Per}(\omega) \otimes \mathbb{Q}$ form a vector space of rank 2 over $K^{\prime}=\mathbb{Q}(\operatorname{tr} \gamma)\left[\mathrm{Mc} 2\right.$, Thm 9.5], and thus $K^{\prime}=K$. By Theorem 1.8, this implies there is a $D>0$ with $\mathbb{Q}(\sqrt{D})=\mathbb{Q}(\sqrt{d})$ such that $(X, \omega) \in G_{D}$. In particular, $(X, \omega)$ generates a Teichmüller curve.

Remark: An open subset of $\Omega G$. Since $\Omega G$ is linear in period coordinates, Theorem 1.9 also holds for the open set of complex parameters $(a, b)$ that determine embedded polygons. In fact, if we apply the action of $\mathrm{GL}_{2}^{+}(\mathbb{R})$ to the resulting forms, then we obtain a dense open subset of $\Omega G$. Figures 6 and 7 show cathedral polygons of this more general type.

\section{References}

[An] S. M. Antonakoudis. Teichmueller spaces and bounded symmetric domains do not mix isometrically. Preprint, 2015. 
[ACGH] E. Arbarello, M. Cornalba, P. A. Griffiths, and J. Harris. Geometry of Algebraic Curves, volume I. Springer-Verlag, 1985.

[AD] M. Artebani and I. Dolgachev. The Hesse pencil of plane cubic curves. Enseign. Math. 55(2009), 235-273.

[Be] F. Berteloot. Une caractérisation géométrique des exemples de Lattès de $\mathbf{P}^{k}$. Bull. Soc. Math. France 129(2001), 175-188.

[BM] I. I. Bouw and M. Möller. Teichmüller curves, triangle groups and Lyapunov exponents. Ann. of Math. 172(2010), 139-185.

[Ca] K. Calta. Veech surfaces and complete periodicity in genus 2. J. Amer. Math. Soc. 17(2004), 871-908.

[Cay] A. Cayley. A memoir on curves of the third order. Philos. Trans. Roy. Soc. London 147(1857), 415-446.

[Cr] L. Cremona. Introduzione ad una theoria geometrica delle curve piane. Mem. Accad. Sci. Inst. Bologna 12(1862), 305-436.

[DH] M. Dabija and M. Jonsson. Algebraic webs invariant under endomorphisms. Publ. Mat. 54(2010), 137-148.

[Dol] I. Dolgachev. Classical Algebraic Geometry. Cambridge University Press, 2012.

[EMM] A. Eskin, M. Mirzakhani, and A. Mohammadi. Isolation, equidistribution, and orbit closures for the $\mathrm{SL}_{2}(\mathbb{R})$ action on moduli space. To appear, Annals of Mathematics.

[Ga] F. Gardiner. Teichmüller Theory and Quadratic Differentials. Wiley Interscience, 1987.

[vG] G. van der Geer. Hilbert Modular Surfaces. Springer-Verlag, 1987.

[Gen] Q. Gendron. The Deligne-Mumford and the incidence variety compactifications of the strata of $\Omega \mathcal{M}_{g}$. Preprint, 2015.

[GH] P. Griffiths and J. Harris. Principles of Algebraic Geometry. Wiley Interscience, 1978.

[Ho] W. P. Hooper. Grid graphs and lattice surfaces. Int. Math. Res. Not. 2013 pages $2657-2698$. 
[Te] M. Teixidor i Bigas. The divisor of curves with a vanishing thetanull. Compositio Math. 66(1988), 15-22.

[KS] R. Kenyon and J. Smillie. Billiards on rational-angled triangles. Comment. Math. Helv. 75(2000), 65-108.

[Lei] C. J. Leininger. On groups generated by two positive multi-twists: Teichmüller curves and Lehmer's number. Geom. Topol. 8(2004), 1301-1359.

[Mas] H. Masur. Ergodic theory of translation surfaces. In Handbook of Dynamical Systems, Vol. 1B, pages 527-547. Elsevier B. V., 2006.

[Mc1] C. McMullen. Billiards and Teichmüller curves on Hilbert modular surfaces. J. Amer. Math. Soc. 16(2003), 857-885.

[Mc2] C. McMullen. Teichmüller geodesics of infinite complexity. Acta Math. 191(2003), 191-223.

[Mc3] C. McMullen. Prym varieties and Teichmüller curves. Duke Math. J. 133(2006), 569-590.

[Mo1] M. Möller. Periodic points on Veech surfaces and the Mordell-Weil group over a Teichmüller curve. Invent. math. 165(2006), 633-649.

[Mo2] M. Möller. Linear manifolds in the moduli space of one-forms. Duke Math. J. 144(2008), 447-487.

[Mo3] M. Möller. Affine groups of flat surfaces. In A. Papadopoulos, editor, Handbook of Teichmüller Theory, volume II, pages 369-387. Eur. Math. Soc., 2009.

[Mu1] R. Mukamel. Fundamental domains and generators for lattice Veech groups. Preprint, 2013.

[Mu2] R. Mukamel. Visualizing the unit ball for the Teichmüller metric. To appear, J. Exp. Math.

[Ro] F. Rong. Lattès maps on $\mathbf{P}^{2}$. J. Math. Pures Appl. 93(2010), 636-650.

[Roy] H. L. Royden. Automorphisms and isometries of Teichmüller space. In Advances in the Theory of Riemann Surfaces, pages 369-384. Princeton University Press, 1971. 
[Sal] G. Salmon. Higher Plane Curves. Hodges, Foster and Figgis, 1879.

[V] W. Veech. Teichmüller curves in moduli space, Eisenstein series and an application to triangular billiards. Invent. math. 97(1989), $553-583$.

[Vo] Ya. B. Vorobets. Plane structures and billiards in rational polygons: the Veech alternative. Russian Math. Surveys 51(1996), 779-817.

[Wr1] A. Wright. Schwarz triangle mappings and Teichmüller curves: the Veech-Ward-Bouw-Möller curves. Geom. Funct. Anal. 23(2013), 776-809.

[Wr2] A. Wright. The field of definition of affine invariant submanifolds of the moduli space of abelian differentials. Geom. Topol. 18(2014), 1323-1341.

[Wr3] A. Wright. Cylinder deformations in orbit closures of translation surfaces. Geom. Topol. 19(2015), 413-438.

[Z] A. Zorich. Flat surfaces. In Frontiers in Number Theory, Physics, and Geometry. I, pages 437-583. Springer, 2006.

Mathematics Department, Harvard University, Cambridge, MA 02138-2901

Mathematics Department, Rice University, Houston, TX 77005

Mathematics Department, Stanford University, Stanford, CA 94305 